\title{
Tools of the trade: \\ Estimating time-varying connectivity patterns from fMRI data
}

\author{
Armin Iraji ${ }^{1}$, Ashkan Faghiri ${ }^{1}$, Noah Lewis ${ }^{1}$, Zening Fu ${ }^{1}$, Srinivas Rachakonda ${ }^{1}$, Vince D. Calhoun ${ }^{1, *}$ \\ ${ }^{I}$ Tri-Institutional Center for Translational Research in Neuroimaging and Data Science (TReNDS), Georgia State University, \\ Georgia Institute of Technology, and Emory University, Atlanta, GA 30303, USA \\ *Correspondence: vcalhoun@gsu.edu (V.D. Calhoun).
}

\begin{abstract}
Given the dynamic nature of the brain, there has always been a motivation to move beyond "static" functional connectivity, which characterizes functional interactions over an extended period of time. Progress in data acquisition and advances in analytical neuroimaging methods now allow us to assess the whole brain's dynamic functional connectivity $(\mathrm{dFC})$ and its network-based analog, dynamic functional network connectivity (dFNC) at the macroscale $(\mathrm{mm})$ using fMRI. This has resulted in the rapid growth of analytical approaches, some of which are very complex, requiring technical expertise that could daunt researchers and neuroscientists. Meanwhile, making real progress toward understanding the association between brain dynamism and brain disorders can only be achieved through research conducted by domain experts, such as neuroscientists and psychiatrists. This article aims to provide a gentle introduction to the application of $\mathrm{dFC}$. We first explain what $\mathrm{dFC}$ is and the circumstances under which it can be used. Next, we review two major categories of analytical approaches to capture dFC. We discuss caveats and considerations in $\mathrm{dFC}$ analysis. Finally, we walk readers through an openly accessible toolbox to capture $\mathrm{dFC}$ properties and briefly review some of the dynamic metrics calculated using this toolbox.
\end{abstract}

\section{Keywords}

dynamic functional connectivity, spatially dynamic, temporally dynamic, spatiotemporally dynamic, fMRI 


\section{Dynamic functional connectivity (time-varying functional patterns)}

It has been suggested that cognition and many mental activities result from the interactions of distributed brain areas (Bressler and Menon, 2010). A local neural assembly, which has its own intrinsic functionality, interacts at the global level with other parts of the brain. However, a major challenge to studying the brain from this point of view is how to best capture functional interactions across the whole brain. An ideal solution would be to evaluate whole-brain dynamic interactions at the neural level; however, imaging at such a scale in humans is not possible at present. Instead, functional imaging techniques can be used to assess the whole-brain functional interactions at a macro-scale $(\mathrm{mm})$ resolution and have yielded information of great value. Functional magnetic resonance imaging (fMRI) measures the blood oxygenation level-dependent (BOLD) signal, a macro-scale proxy for average neural activity, which allows simultaneous investigation of the functional localization and interactions between brain regions. Most commonly, the entire fMRI scan is used to calculate the average functional connectivity (FC), a method known as static functional connectivity $(\mathrm{sFC})$. However, spontaneous brain activity is rich with dynamic properties which are disregarded in such a method. Therefore, research into whole brain dynamic functional connectivity (dFC) has become a burgeoning field of study since initial work on the topic (Sakoglu et al., 2010; Lurie et al., 2020). Although one can distinguish between approaches that leverage the time-varying signal and those that more explicitly model/capture dynamics over time (Lurie et al., 2020), we refer to both as dFC for convenience. dFC is defined as time-varying functional connectivity and contains information regarding the temporal reconfiguration of functional entities (also called sources). $\mathrm{dFC}$ studies aim to evaluate how the interactions between functional sources change over time. We can define a functional source as a group of temporally synchronized neural assemblies, which present similar functionality within a given dataset (Iraji et al., 2020). Fixed anatomical locations are convenient representations of these sources, assuming all voxels within a predefined anatomical location have the same functional profile and are the same across individuals. More advanced approaches, such as dynamic functional network connectivity ( $\mathrm{dFNC}$ ), leverage the data itself to estimate the sources and study dFC (Jafri et al., 2008; Allen et al., 2011; Calhoun and de Lacy, 2017).

It should be noted that because fMRI is an indirect measurement of neural activity, fluctuations in FC estimated by fMRI are also indirect representations of dFC. There is ongoing discussion regarding how well these fluctuations capture the underlying brain dynamism, but previous studies provide a significant amount of evidence to support the potential relationship between the fluctuations in FC obtained from 
fMRI and neural dynamics in the brain (for review see (Lurie et al., 2020)). For instance, simultaneous fMRI and EEG imaging studies show that fluctuations in FC obtained from rsfMRI are associated with electrophysiological signatures of EEG (Chang et al., 2013; Allen et al., 2018). A comparison between the hemodynamic signal and neuronal calcium signal also provides strong evidence that temporal fluctuations in hemodynamic FC are related to brain dynamics (Matsui et al., 2019). Thus, to simplify notation in the remainder of this paper, we refer to time-varying FC estimates from fMRI as dFC.

Studies have also evaluated the replicability and reliability of dFC properties captured by fMRI. Previous researchers used a large dataset ( 7500 subjects) collected from different sites and identified replicable $\mathrm{dFC}$ patterns, which are robust against variations in data quality and analysis methods (Abrol et al., 2017). Test-retest reliability analyses show the presence of reliable dynamic patterns (Choe et al., 2017; Zhang et al., 2018a). Similar studies also identify reliable and reproducible intersession (intervals of two days and one week) dFC patterns (Yang et al., 2014; Smith et al., 2018). Shi et al. reported robust findings of the association between $\mathrm{dFC}$ and subjective well-being across two independent datasets and different analysis parameters, which suggests $\mathrm{dFC}$ is involved in self-focused processing, emotional regulation, and the cognitive control process (Shi et al., 2018). dFC also helps to index mental states dictated by a multitask paradigm and can differentiate between task-induced cognitive processes (Gonzalez-Castillo et $a l ., 2015)$. dFC patterns of the salience network and the posteromedial cortex are related to individual differences in cognitive flexibility and categorization ability (Yang et al., 2014; Chen et al., 2016). Using a continuous auditory detection task, Sadaghiani et al. showed that dFC features before the auditory stimulus could predict whether the audio was recognized or not, and $\mathrm{dFC}$ patterns after stimulation were also significantly different between the two scenarios (Sadaghiani et al., 2015). Madhyastha et al. show that $\mathrm{dFC}$ within the dorsal attention network and frontoparietal network (FPN) predicts attention task performance (Madhyastha et al., 2015). The dFC variability (see Table 1 for the definition of different dFC measures) between the default mode network (DMN) and FPN is shown to be related to cognitive performance (Douw et al., 2016). Compared to low trait mindfulness individuals, high trait mindfulness individuals show a higher level of transitions between brain $\mathrm{dFC}$ states and spend more time in one $\mathrm{dFC}$ state associated with task-readiness (Lim et al., 2018). Marusak et al. similarly found that dFC (but not $\mathrm{sFC}$ ) is related to mindfulness in youths such that more mindful youths have a higher level of transitions between dFC states (Marusak et al., 2018). Additionally, Cabral et al. (Cabral et al., 2017) report a relationship between cognitive performance in older adults and slow switching between dFC states. dFC properties appear to be related to age in the early years as well. Young adults, compared to children, have 
decreased $\mathrm{dFC}$ strength between the DMN and other brain networks, and tend to spend more time in one dFC state with a pattern similar to sFC (Cai et al., 2018). Interestingly, regions with different cognitive and processing demands represent different levels of dynamism. The brain networks/areas that are known to be involved in higher cognitive processing show a higher level of dynamism measured by dFC than networks/areas engaged in primary processing (Zalesky et al., 2014; Chen et al., 2016; Iraji et al., 2019b). At the network level, networks that are engaged in a wide range of cognitive functions, such as the FPN, seem to have the highest level of dynamism (Zalesky et al., 2014; Iraji et al., 2019b). The relevance of $\mathrm{dFC}$ is also supported by other studies that evaluate the relationship between $\mathrm{dFC}$ properties and biological features such as age, gender, and cognitive processes (Thompson et al., 2013a; Leonardi et al., 2014; Elton and Gao, 2015; Hutchison and Morton, 2015; Qin et al., 2015; Yaesoubi et al., 2015a; Yaesoubi et al., 2015b; Shine et al., 2016a; Shine et al., 2016b; Kucyi et al., 2017; Kucyi, 2018).

The findings mentioned above support the potential neurophysiological relevance of $\mathrm{dFC}$ and the benefit of studying dFC properties to elucidate the brain function. One key future research direction is the use of cognitive/affective tasks to determine the specific dFC properties of networks/areas which are associated with certain cognitive processing and mental states.

$\mathrm{dFC}$ analysis also has the potential to improve the understanding of impaired brains and the functional alterations caused by brain disorders. Compared to static analysis, dFC analysis provides additional information about the temporal profile of brain function and its changes in disorders. dFC analysis can detect nuanced alterations that are averaged out in static analysis (Calhoun et al., 2014; Iraji et al., 2019a). In addition, $\mathrm{dFC}$ has been shown to explain some of the inconsistencies in sFC findings (Iraji et al., 2019a). dFC studies also suggest promising research directions in neurological and psychiatric disorders (see Considerations and caveats for further details) (Calhoun et al., 2014). Towards this goal, researchers have started using different methods and metrics to investigate the characteristics of dFC in various brain disorders.

Among various brain disorders, schizophrenia (SZ) has been one of the most widely studied via dFC. Patients with SZ show higher dynamic global efficiency across the brain (Sun et al., 2019). Damaraju et al. reported the most substantial differences between SZ and control groups in dFC states with strong negative cortical-subcortical connectivity and strong positive connectivity between sensory networks (Damaraju et al., 2014). Patients with SZ spend less time in a dFC state with strong connectivity, and the strengths of $\mathrm{dFC}$ between subcortical and sensory networks are weaker in these patients (Damaraju et al., 
2014). Spatial dynamics studies also have revealed weaker dFC strength (transient hypoconnectivity) within particular networks (Iraji et al., 2019a; Iraji et al., 2019b) in SZ. It has been shown that the decrease in $\mathrm{dFC}$ strength is accompanied by higher fluctuations in dFC between brain regions (Yue et al., 2018) and within and between certain brain networks (Ma et al., 2014; Iraji et al., 2019a) in patients with SZ. Studies also show frequency-specific dFC alterations in patients with SZ (Yaesoubi et al., 2017; Zhang et al., 2018b; Faghiri et al., 2019). The atypical dFC patterns in patients with SZ may be related to disease traits such as reduced cognitive performance. The amygdala-medial prefrontal cortex (mPFC) dFC variability is shown to be positively correlated with the total PANNS (positive and negative symptom scale) score and negatively correlated with information processing efficiency indexed by the digit symbol coding task (Yue et al., 2018). The correlation between the energy index (spatiotemporal uniformity) of the subcortical domain and the attention/vigilance computerized multiphasic interactive neurocognitive dualdisplay system (CMINDS) cognitive domains was disrupted in SZ patients (Iraji et al., 2019a). The correlation between activity and $\mathrm{dFC}$ of the cuneus was associated with the working memory domain of CMINDS in patients with SZ and controls (Fu et al., 2018).

In another clinical condition, a study found that individuals with major depressive disorder (MDD) more time in a state with weak functional connectivity strength across the brain (weakly-connected dFC state) associated with self-focused thinking (Zhi et al., 2018). Atypical dFC patterns were significantly correlated with both depressive symptoms and cognitive performance (Zhi et al., 2018). Qiu et al. studied the dFC of the amygdala's subregions in untreated individuals with first-episode MDD and found a decrease in the dFC strength between specific amygdala subregions and several regions within the limbiccortical-striato-pallido-thalamic circuit (Qiu et al., 2018). Additionally, they showed that the age of MDD onset correlates with the dFC strength between the left centromedial subregion of the amygdala and the brainstem (Qiu et al., 2018). In a different study, MDD patients show decreased variability of dFC between the DMN and the PFN (Demirtas et al., 2016).

Alterations in $\mathrm{dFC}$ features have been observed in patients with dementia as well. Change in anteriorposterior $\mathrm{dFC}$ of the brain was associated with declined episodic memory performance in the elderly (Quevenco et al., 2017). Patients with Alzheimer's disease (AD) showed higher and lower dwell time in $\mathrm{dFC}$ state with strong the anterior and posterior DMN, respectively (Jones et al., 2012). Patients with AD also show alteration in whole-brain dFC (Cordova-Palomera et al., 2017; Schumacher et al., 2019) and spend more time in the weakly-connected dFC state (Schumacher et al., 2019). Patients with AD show 
both common and distinct dFC patterns with patients who have subcortical ischemic vascular disease (SIVD), while the clinical features and symptoms between these two disorders can sometimes be difficult to distinguish (Fu et al., 2019a). In autism spectrum disorder (ASD), alterations in dFC patterns were observed both within the DMN and between the DMN and networks involved in higher cognitive processing, including the PFN and the cingulo-opercular network (de Lacy et al., 2017). Fu et al. found that individuals with ASD show a transient increase in $\mathrm{dFC}$ strength between hypothalamus/subthalamus and sensory networks, as well as alterations in several meta-state metrics including the number of metastates and total traveling distance ( $\mathrm{Fu}$ et al., 2019b). Interestingly, these atypical dFC patterns are significantly associated with the total autism diagnostic observation schedule (ADOS) score. Guo et al. (Guo et al., 2019) conducted a study to test the role of the right anterior insula in neurophysiological mechanisms underlying the social impairments of ASD. Their analyses show a decrease in dFC strength between the right anterior insula and the regions associated with the DMN, including the ventral medial prefrontal cortex and posterior cingulate cortex (PCC) dFC (Guo et al., 2019). Aligned with these findings, Rashid et al. found that children with high levels of autistic traits and ASD diagnoses linger more in the weakly-connected dFC state and less in the dFC state with strong DMN connectivity (Rashid et al., 2018). It has been suggested that decreased dFC variability of the PCC is related to the role of the DMN in the social-cognitive deficits of ASD. The lower dFC variability between the PCC and sensorimotor cortex was correlated with deficits in social motivation and social relating in ASD individuals (He et al., 2018). Harlalka et al. reported positive correlations between ADOS scores and dFC variability, particularly in the DMN connections (Harlalka et al., 2019). Jones et al. suggested the dFC of the DMN might be related to the cognitive deterioration in individuals at risk for Alzheimer's dementia (Jones et al., 2012).

The dFC of the DMN and its associated areas were also associated with cognition in other populations. In (left) temporal lobe epilepsy, the lower dFC variability of the PCC was related to disturbed verbal memory functioning (Douw et al., 2015). The $\mathrm{dFC}$ of the DMN and memory functioning were positively correlated in Parkinson's disease (PD) patients (Engels et al., 2018). PD patients also show a loss of specificity of $\mathrm{dFC}$ in putaminal subunits with the exception of the caudal middle frontal gyrus (Liu et al., 2018). Changes in the dFC strength of the putamen subunits (particularly anterior subunits) are shown to be correlated with the unified Parkinson's disease rating scale III (UPDRS III), and joint dFC features (strength and variability) were able to predict UPDRS III and Montreal cognitive assessment (MoCA) scores (Liu et al., 2018). Furthermore, PD patients with mild cognitive impairment (MCI) showed a significantly higher number of transitions between $\mathrm{dFC}$ states and reduced dwell time in the weakly-connected state compared 
to healthy controls, while there was no difference observed for PD patients with normal cognition. DíezCirarda et al. reported atypical dFC patterns in PD patients, mostly between regions associated with somatomotor and regions associated with cognitive-control (Díez-Cirarda et al., 2018).

Alterations in dFC states were also observed in mild traumatic brain injury (mTBI) patients (Vergara et al., 2018; Hou et al., 2019; van der Horn et al., 2019) and had better discriminatory power than sFC (Vergara et al., 2018). In relapsing-remitting multiple sclerosis (RRMS), dorsal and ventral attention networks displayed lower within-network $\mathrm{dFC}$ and higher between-network $\mathrm{dFC}$, and the $\mathrm{dFC}$ alterations were linked to white matter lesion damage (Huang et al., 2019). RRMS subjects were reported to have overall reduced global $\mathrm{dFC}$ but increased interhemispheric $\mathrm{dFC}$ (possibly to compensate for reduced global dFC) (Lin et al., 2018). Better executive functions in RRMS patients were associated with higher $\mathrm{dFC}$ (Lin et al., 2018). d'Ambrosio et al. reported that, compared to cognitively preserved MS patients, cognitively impaired MS (CI-MS) patients have lower dwell time in highly-connected states and reduced subcortical-DMN dFC in a weakly-connected state (d'Ambrosio et al., 2019). Meta-state analysis (see Window-based approaches (WBAs) for definition) also show reduced dynamism in CI-MS patients (d'Ambrosio et al., 2019). Atypical dFC patterns present in other cohorts and brain disorders, including migraine (Tu et al., 2019), stroke (Chen et al., 2018), epilepsy (Douw et al., 2015; Liu et al., 2017; KlugahBrown et al., 2019), attention deficit hyperactivity disorder (ADHD) (Ou et al., 2014; de Lacy and Calhoun, 2019), post-traumatic stress disorder (Li et al., 2014; Jin et al., 2017), frontotemporal dementia (Premi et al., 2019), and Lewy body dementia (Schumacher et al., 2019).

Despite the rising interest and great potential of $\mathrm{dFC}$ in various circumstances, the application of $\mathrm{dFC}$ is still not a baseline tool for neuroscientists. One main reason is the rapidly growing pool of analytical approaches. In the following sections, we provide a basic summary of the most common analytical approaches and a straightforward tool to study dFC.

\section{Analytic approaches to study dFC}

In general, $\mathrm{dFC}$ studies probe the dynamic properties of the brain via variations in $\mathrm{FC}$ estimations over time. A dFC study can evaluate changes in spatial patterns of functional sources over time (also known as spatial dynamics) and/or variations in their activity profiles of sources over time (which is called temporal dynamics) (Figure 1) (Iraji et al., 2020). Several analytical techniques have been proposed to capture and evaluate $\mathrm{dFC}$ using fMRI data (Figure 2). Here, we review essential concepts and terms behind two major 
nonexclusive categories, "window-based approaches (WBAs)" and "event detection approaches (EDAs)." There are several technical reviews on different approaches of these two categories, as well as other categories of methods, such as those that use dynamic modeling techniques or temporal sequence information (Hutchison et al., 2013; Calhoun et al., 2014; Keilholz et al., 2017; Preti et al., 2017).

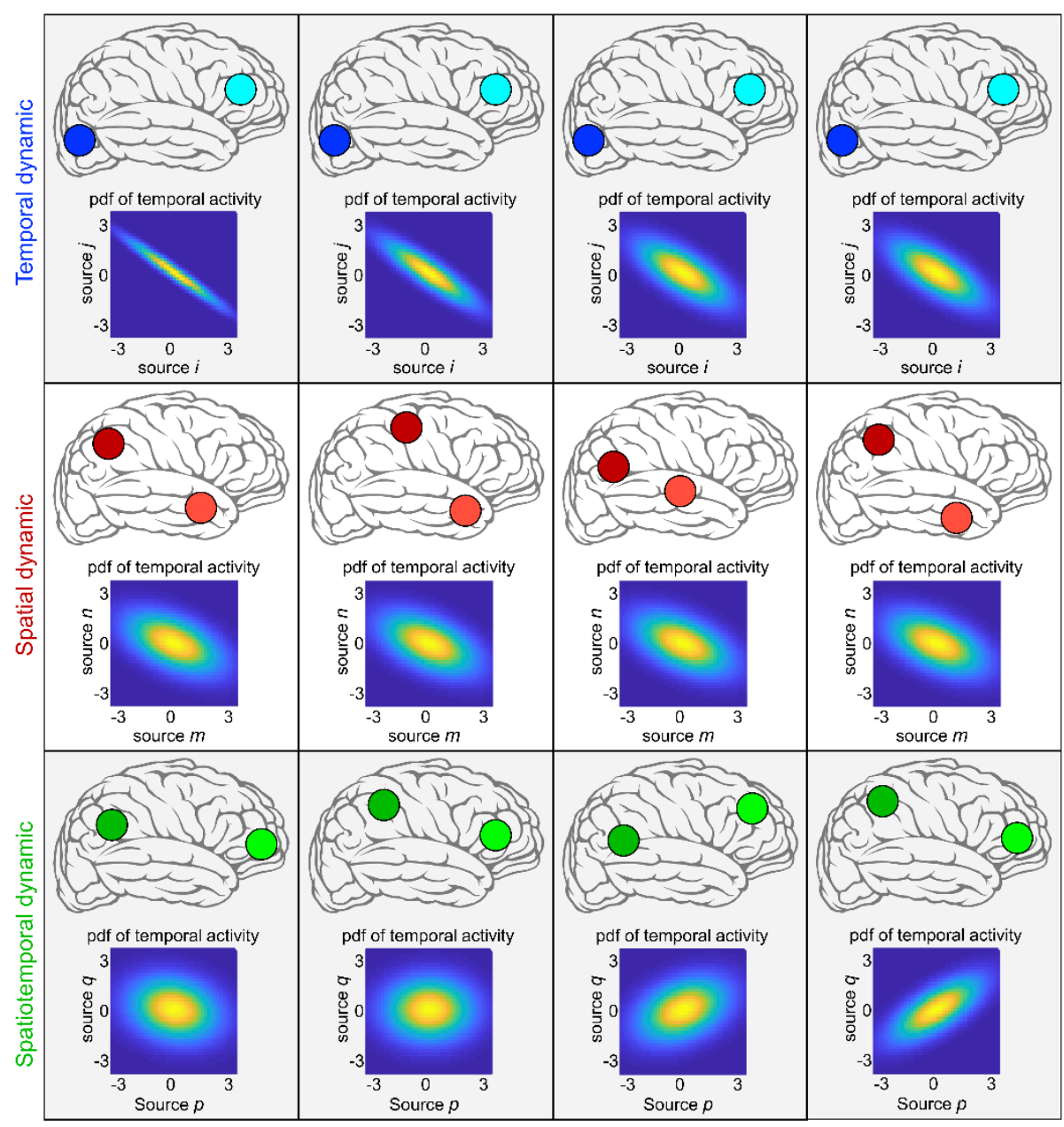

Figure 1. Example of temporal, spatial, and spatiotemporal dynamic with a scenario that the brain has only two functional sources. The brain is temporally dynamic if the temporal coupling between the temporal activity of sources varies over time. The brain is spatially dynamic if the spatial properties of sources change over time (e.g., translations of sources in space). If functional sources hold both spatially and temporally dynamic properties, it is spatiotemporally dynamic.

FC is estimated by calculating the statistical association between measured brain signals, commonly between different spatial localities (nodes) (Figure 2A). A node can be a voxel, an anatomical region/seed, or computed from the fMRI data itself, e.g., an intrinsic connectivity network (ICN). Functional homogeneity within a node is the crucial factor in defining nodes. For instance, when we use anatomical regions as nodes, we should verify that the voxels within a node must have more similar time courses than voxels from different nodes. To ensure functional homogeneity, we can use data-driven approaches like 
independent component analysis (ICA) to estimate the ICNs as nodes (Calhoun and Adali, 2012). ICA is a multivariate approach that simultaneously estimates the spatial patterns and activity profiles (time courses) of ICNs. For simplicity, we assume FC is calculated using Pearson correlation, but other metrics such as coherence are equally valid and can be used to capture additional information (Yaesoubi et al., 2015a; Salman et al., 2019).

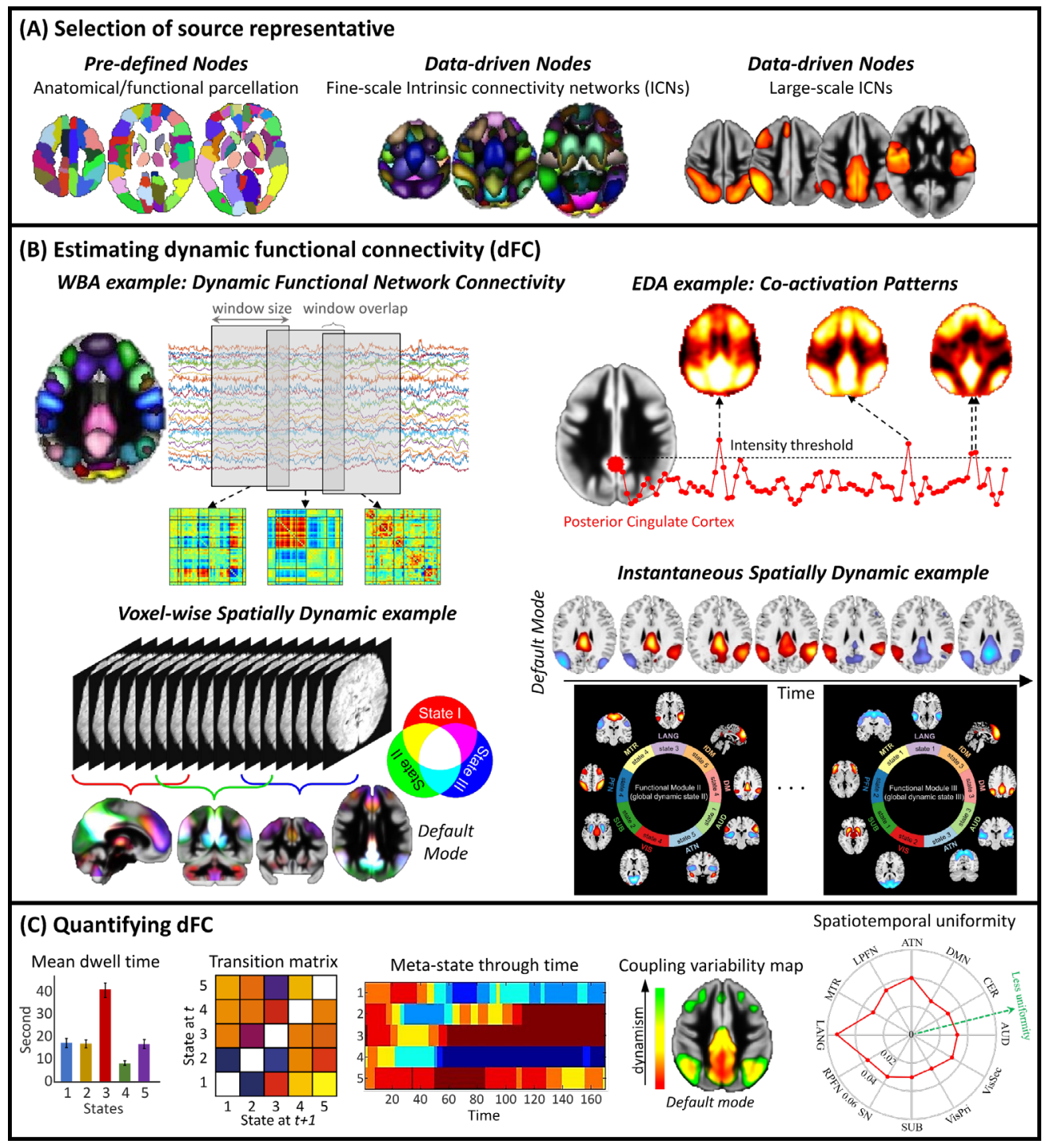

Figure 2. Cartoon examples of the analysis pipeline dynamic functional connectivity (dFC) analysis. (A) we first select nodes (proxies for spatial locations of sources) to calculate functional connectivity between sources of interests. (B) we use a dFC estimator approach to calculate dFC between selected nodes. Different estimators measure different dynamic properties (temporal dynamic or spatial dynamic). WBA: Window-based approaches, EDA: Event detection approaches. (C) After estimating dFC, various techniques can be used to quantify $\mathrm{dFC}$ and evaluate dynamic properties. 


\section{Window-based approaches (WBAs)}

The substantial similarity with conventional FC (i.e., sFC) and ease of use make the applications of WBAs to study $\mathrm{dFC}$ and the interpretation of their findings straightforward. Window-based approaches (commonly known as sliding-window approaches), in simple terms, estimate conventional FC for durations larger than the sampling rate of the acquired data (Sakoglu et al., 2010). The time courses are divided into short segments (time windows), and FC is calculated for each time window. This results in a series of windowed-FC over time (FC as a function of time) that contains $\mathrm{dFC}$ information. WBAs require defining time window and the node(s) of interest before calculating windowed-FC (Figure 2B).

Time windows can have different sizes, shapes, and window overlaps, but these parameters commonly remain constant throughout a study. The best choice of these parameters is unknown and can be different depending on the available data and the goals of studies. However, we can make a general recommendation from previous literature. For the window shape, the most common choice is tapered windows (Allen et al., 2014; Leonardi and Van De Ville, 2015). For the window overlap, windows with zero overlap or and maximum overlap (all time points except one time point are shared between consecutive windows) are the two most common choices. The most important aspect of time windows to capture $\mathrm{dFC}$ is the window size. Very small window sizes may not have enough information to estimate $\mathrm{dFC}$ robustly and thus introduce spurious fluctuations while large window sizes may smooth out the dynamic properties and fail to capture dynamic properties (Fu et al., 2014; Vergara et al., 2019). Ideally, we should choose window sizes that match the timescale of underlying brain dynamism; however, there is no prior information about the underlying dFC profile. At the same time, studies suggest WBAs can distinguish underlying mental states even using window sizes substantially different from the duration of the underlying cognitive processes. For instance, Gonzalez-Castillo et al. (Gonzalez-Castillo et al., 2015) modulate mental states using a series of well-defined cognitive tasks. While the optimal window size to match the transition between mental states was 180 seconds, window sizes as short as 22.5 seconds accurately track mental states. A recommended window size is between 30 to 60 seconds (Leonardi and Van De Ville, 2015; Zalesky and Breakspear, 2015).

Meanwhile, several approaches have been proposed to circumvent the selection of window size. One may use adaptive window size approaches to match window size with the underlying brain dynamics by estimating local stationarity or change points (Fu et al., 2014; Xu and Lindquist, 2015; Jeong et al., 2016; Jin et al., 2017). One can also explore dFC at different frequencies which is similar to adapting the window 
size to the frequency scale (Chang and Glover, 2010; Yaesoubi et al., 2015a). Another solution is to estimate FC for single time points (instantaneous) FC and reduce or even eliminate the need of choosing a window (Thompson et al., 2018; Yaesoubi et al., 2018; Faghiri et al., 2019; Iraji et al., 2019b; Faghiri et al., 2020). The next step after defining our window parameters is to select the node(s) of interest. Commonly, we select the nodes from across the whole brain to summarize the whole brain dFC as perwindow FC matrices for the given nodes (Allen et al., 2014). We can also focus on the dFC of a specific set of nodes based on the hypothesis of a study (Yang et al., 2014). Another option is to calculate the FC of each source with every voxel of the brain for every time window to provide a detailed whole-brain, comprehensive map of the $\mathrm{dFC}$ of each source (Iraji et al., 2019a).

Once dFC is calculated via WBAs, various techniques can be used to quantify dFC and evaluate dynamic properties (Figure 2C) (see "Implementing a dFC study: A GIFT walkthrough" for some examples of metrics to quantify $\mathrm{dFC}$ properties). One standard procedure is to determine distinct and recurring dynamic patterns such as $\mathrm{dFC}$ (meta-)states and calculate $\mathrm{dFC}$ properties by assessing the temporal profiles of (meta-)states. dFC states are a set of distinct FC patterns, which are commonly identified by grouping windowed-FC using k-means clustering. In this way, each window is assigned to one state (Allen et al., 2014). Meta-state analysis, on the other hand, assumes windowed-FCs are a combination of meta-states with continuous contributions over time (Miller et al., 2016). Indeed, there is a close relationship between states and meta-states. Meta-states are equal to states if only one of them is present at any given time (power of all but one of them is zero). It is worth mentioning that both terms, states and meta-states, have been used for different purposes in literature. For instance, while in dFC studies, meta-state refers to an instantaneous coordinate of the brain in the state-space (Miller et al., 2016), it has also been used to refer to FC patterns which recur across sessions (Shine et al., 2016b). As the actual number of (meta-) states of the brain is unknown, it needs to be estimated using different techniques and criteria. Event detection approaches (see Event detection approaches (EDA)) carry the same limitations as they estimate dynamic states in a similar manner. (Meta-) states can then be evaluated by different metrics such as dwell time, fraction rate, the number of transitions (switching), total traveling distance, the total number of (meta-) states met during the length of scan, and community/modularity of states. We can also directly evaluate the $\mathrm{dFC}$ properties from windowed-FC for instance by calculating functional connectivity variation over time (dFC variability) and spatiotemporal transition matrix. 


\section{Event detection approaches (EDA)}

In a nutshell, EDAs identify dynamic states by grouping time points based on the similarity in the amplitude of BOLD signals (or its derivations) from a subset of the brain's regions or the entire brain (Figure 2B). The idea behind the EDA can be explained as follows: a group of functionally connected regions (neural assemblies) activates together in response to either internal or external events (stimuli), which results in a momentarily increase in their BOLD signal amplitude. Assuming each dynamic state represents a distinct pattern of spontaneous events, we can obtain brain dynamic states by identifying different (co-)activation patterns in the time series. Note that this is a simplification of the idea behind EDAs and their relationship with the dFC states. The EDAs were initially developed based on the hypothesis that spontaneous BOLD signal originates from infrequent (i.e., sparse in time) neuronal events, such as large-scale neuronal avalanching activity (Chialvo, 2010; Tagliazucchi et al., 2011; Tagliazucchi et al., 2012). EDAs commonly consist of three steps: 1) detecting the time points in which neural-related events occur, 2) grouping the selected time points to identify different dynamic states, 3) quantifying dynamic properties using different metrics (similar to the examples discussed WBAs).

Several methods have been developed to detect the time points in which spontaneous, infrequent events occur. The simplest and most common category of time point detection methods leverages the amplitude of the BOLD signal. Point process analysis (PPA) (Tagliazucchi et al., 2012) and co-activation patterns (CAPs) (Liu et al., 2013) commonly use this category of time point detection methods. Two familiar procedures to select time points from the amplitude of BOLD signal are 1) choosing time points which pass a threshold value (e.g., above one standard deviation of the time series) (Tagliazucchi et al., 2011; Tagliazucchi et al., 2012; Liu and Duyn, 2013) and 2) selecting time points which are the local maxima/minima of the time series (Tagliazucchi et al., 2016). Another category of time point detection methods uses deconvolution techniques, which was previously applied to task-based fMRI studies (Gitelman et al., 2003). A hemodynamic model is used to deconvolve the BOLD signals and resolve them into a set of sparse, event-related time points. Paradigm free mapping (PFM) (Caballero Gaudes et al., 2013) and total activation (TA) (Karahanoglu et al., 2013) are some examples of time point detection methods that use the hemodynamic deconvolution technique. In the same category, the innovation-driven CAPs (iCAPs) approach suggests applying temporal derivatives to the deconvolved BOLD signal to capture transient information (identify regions with a simultaneous increase or decrease) (Karahanoglu and Van De Ville, 2015). While standard EDAs are based on the assumption of sparse events, a study may 
disregard this fundamental assumption and consider all time points for the second step of the analysis (Liu et al., 2013). In the second step, we divide the selected (event-related) time points into multiple groups based on the similarity between their spatial patterns (commonly using k-means clustering). Each group (cluster) represents a dynamic state and consists of time points with a similar co-activation (spatial) pattern, which is distinct from other groups. Subsequent thresholding is commonly used to identify regions associated with each state. The second step is similar to how WBAs identify dynamic states, but instead of using the similarity between connectivity patterns, EDAs use the similarity between co-activation patterns. Interestingly, the results of EDAs resemble the spatial patterns of well-known FC patterns, such as large-scale networks, which further highlights the similarity between co-activation and FC patterns. This resemblance is somewhat expected as when two regions are co-activated, they are also co-varying over time which fits the definition of statistical dependency and FC. Finally, like the third step of WBAs, dynamic properties and the timing of dynamic states can be quantified using various metrics such as dwell time, transition probability, and the occurrence rate of dynamic states.

Instead of identifying recurring time points, some EDAs focus on identifying recurring patterns of sequences of time points. In other words, these approaches are interested in finding a particular temporal sequence that repeats over time. For example, quasi-periodic patterns are a sequence of consecutive time points that recur during a scan (Majeed et al., 2011). It is worth mentioning that the idea behind these approaches is closely related to propagating waves observed in other imaging modalities (Matsui et al., 2016; Muller et al., 2018). There are also other approaches that detect dynamic states by characterizing temporal sequence information at the cost of additional computations and stricter assumptions, such as specific state-space models (hidden Markov models) (Eavani et al., 2013; Vidaurre et al., 2017).

While EDAs can be used to evaluate dFC properties, like any other approach, EDA methods come with assumptions and limitations. For instance, the choices of threshold or deconvolution parameters can significantly affect the sensitivity of EDAs to detect the time points of events and therefore alter results. Another major issue is sensitivity to noise. Because the fMRI signal has a low contrast to noise ratio (CNR), using individual time points makes EDAs significantly susceptible to noise. As a result, detecting time points of events and allocating time points to dynamic states can be inaccurate due to low SNR. For instance, noise contamination can influence local maxima/minima or alter those time points that survive thresholding, and thus makes the selection of event-related time points inaccurate. Deconvolution techniques are also inherently sensitive to noise and are constrained by the specific assumptions of their 
HRF model. The susceptibility to noise becomes more concerning when we study the temporal patterns of dFC. For instance, when we use temporal ordering information to identify dynamic states or when we quantify $\mathrm{dFC}$ using the temporally-dependent measures like dwell time, fraction rate, etc. Moreover, EDAs commonly use anatomical regions as nodes to detect events. This demands additional prespecified parameters. Using fixed anatomical regions makes EDAs susceptible to functional inhomogeneity within nodes and disregards inter- and intra-subject spatial variability.

\section{Considerations and caveats}

$\mathrm{dFC}$ can occur at different time scales, from milliseconds to the entire life span, and fMRI provides an excellent opportunity to non-invasively study whole-brain dynamics in spatial and temporal resolutions simultaneously, which currently cannot be achieved using other imaging modalities. More specifically, fMRI can capture hemodynamic dFC that occurs on the order of seconds with spatial resolution in the order of a millimeter. While most fMRI dFC studies use data that is collected in a temporal resolution of 0.7-3 seconds, higher sub-second temporal resolutions can be significantly beneficial. The advantageous of sub-second resolutions include: 1) capturing higher-frequency information of $\mathrm{dFC}$; 2) improving specificity and robustness of findings by providing more data for any given temporal scale (e.g. in sliding window); 3) increasing the sensitivity to identify dynamic states and their onset, which is especially critical for EDAs; 4) more accurately quantifying dFC properties (particularly those measuring temporal profile); and 5) improving efficacy of noise-reduction techniques such as reducing the effect of aliasing.

$\mathrm{dFC}$ is intertwined with many temporal factors such as vigilance, sleep state and arousal states, maturation, aging, and learning experiences (Lurie et al., 2020). While a substantial body of evidence supports the relationship between $\mathrm{dFC}$ and neural communication, other mechanisms such as physiology, metabolism, autonomic activities, and neurovascular coupling also modulate $\mathrm{dFC}$ patterns. These are important factors to consider when we evaluate $\mathrm{dFC}$ using the BOLD signal (for prior review see (Thompson, 2018)). There is a large body of research on what portion (if any) of changes in FC measured in the BOLD signal is related to brain dynamism (Thompson, 2018), and several methods have been developed to evaluate the significance of various FC measurements against different null hypotheses (Bassett et al., 2013; Lindquist et al., 2014; Hindriks et al., 2016; Laumann et al., 2017). However, one should keep in mind any null model only test the presence of a specific type of $\mathrm{dFC}$ properties, and the result of a statistical test does 
not guarantee the presence or absence of dFC (Miller et al., 2018). Hypothesis-driven studies should be conducted to understand the neural basis and mechanisms of dFC estimated by fMRI.

Furthermore, WBAs and EDAs are neither mutually exclusive nor collectively exhaustive as some analytical approaches can be categorized as both and there are approaches that cannot fit into either of these categories. However, WBAs and EDAs are the most established and verified categories which have straightforward applications in clinical and research environments. Other categorizations such as spatially vs. temporally dynamic approaches, model-based vs. data-driven techniques, and univariate vs. multivariate analyses can provide a more complete picture of existing approaches.

The BOLD signal is an indirect measurement of neural activities and significantly contaminated with many so-called non-neural signals such as motion, heart rate, and respiration. Therefore, it is important to take measures to remove or model the spurious fluctuations and confounding factors from the BOLD signal. At the same time, some of these signals, such as heart rate and motion, are physiological changes associated with neural processes. This impacts the effectiveness of the noise reduction approaches to minimize the contribution of spurious fluctuations, particularly in the absence of ground truth. Therefore, extra care is needed when performing noise-reduction techniques and cleaning procedures to avoid removing meaningful, neural-related information. For instance, although global signal regression, a preprocessing procedure, is shown to improve the relationship between the $\mathrm{AFC}$ of BOLD signal and the dynamic changes in simultaneously recorded local field potentials (LFP) (Thompson et al., 2013b), it may have a negative impact on the reliability dFC analysis (Smith et al., 2018) and cause heterogeneous changes in dFC across the brain (Xu et al., 2018). Regardless of these debates, several pre- and postprocessing procedures, including despiking, filtering, and nuisance-regression, have been recommended as a trade-off to minimize the impact of spurious fluctuations and statistical uncertainty (Hahamy et al., 2014). However there are still many choices to optimize (e.g. filter early or late, etc.) (Vergara et al., 2017).

Finally, while dFC analysis explains specific inconsistencies in SFC findings, the addition of the timevarying properties comes with its own complexities. Furthermore, different analytical approaches use different modeling techniques which might, therefore, capture different aspects of dFC. Some assumptions and limitations of analytical frameworks such as overlooking the inter- and intra-subject spatial variations also lead to inconsistencies and significantly impact the validity of the results. Acquisition parameters and data quality such as low SNR, low temporal resolution, or collecting a short segment of data (short scan 
time) are other components associated with inconsistencies in dFC findings. Furthermore, brain dynamism is unconstrained in nature, and individuals during scans report a variety of different mental activities such as daydreaming, recalling events, planning, dreaming, etc. It is important to consider this when trying to replicate $\mathrm{dFN}$ results, for example, it would be unlikely to replicate the same timing for the above events, however this is probably not the ideal goal for a replication study of dFC.

\section{Concluding remarks}

$\mathrm{dFC}$ analysis using fMRI is still a very active area of development, but it is quickly turning into a critical element of brain research because it provides exceptional opportunity to study brain dynamism and its relationship with different mental states, cognitive conditions, and disorders. In this manuscript, we review some research on the potential association of fMRI-dFC with cognitive demands and behavioral performance. We also present examples of how $\mathrm{dFC}$ patterns are disrupted in various brain disorders and the relationship of atypical $\mathrm{dFC}$ patterns with both cognitive impairments and the outcomes of the disorders (for reviews see (Calhoun and Adali, 2016; Keilholz et al., 2017; Cohen, 2018; Thompson, 2018; Lurie et al., 2020)). Alterations in dFC patterns across a wide range of conditions have been observed even in the absence of $\mathrm{sFC}$ differences. One proposition is that each $\mathrm{sFC}$ pattern is an average of a set of $\mathrm{dFC}$ patterns and therefore smooths out the nuanced differences (Iraji et al., 2020). The dFC patterns are capable of encoding variations across conditions that are more transient than those captured by conventional sFC analyses. Considering the dynamic nature of the brain, the dFC information obtained from fMRI might be a crucial piece in providing a more thorough understanding of the brain's function and characterizing neurological and psychiatric disorders. Quantifying the spatial and temporal dynamics of the brain opens more opportunities to study the brain through a window of dynamism and help answer some of the most compelling questions in cognitive and affective neuroscience. However, this goal requires a hypothesis-driven, carefully designed study and contributions from neuroscientists. Moving forward, dFC research benefits from careful design studies, which allows researchers to understand the mechanisms underlying dynamism, identify neural etiology, elucidate the mechanisms of healthy cognition, investigate individual differences in cognition, and probe $\mathrm{dFC}$ alterations and disruptions in the brain illnesses. This can potentially lead to identifying imaging-based clinical biomarkers for early diagnosis and disease treatments. We encourage researchers who are new to dFC analysis to become more familiar with different analytical steps and limitations of techniques in each step. We also emphasize the 
importance of inter- and inter-subject variability and the need to consider spatial dynamic properties in future studies (Iraji et al., 2020).

\section{Implementing a dFC study: A GIFT walkthrough}

In this section, we provide a brief overview of the $\mathrm{dFC}$ options available in the GIFT toolbox and provide a quick walkthrough to facilitate the application of dFNC analyses for those new to this field. The GIFT toolbox and manual can be found at https://trendscenter.org/software/gift/. GIFT is a MATLAB toolbox which can be used standalone (i.e., a complied version without a MATLAB license) or with MATLAB. It also includes a python interface (giftpy) and is also released as a (docker) containerized tool which also does not require a MATLAB license. GIFT consists of a wide range of independent component analysis (ICA), allowing group inferences from the fMRI data. It also contains several other toolboxes, such as the dynamic functional connectivity toolbox to study brain dynamism and the Mancovan toolbox to determine the significant covariates using multivariate analysis of covariance and a stepwise regression model as well as univariate testing. The resulting covariates found out to be significant can be used in a univariate framework. The noise cloud toolbox uses both spatial and temporal characteristics of independent components to automatically identify noise/artifact components from the specified components after an initial training process. This can also be used to clean fMRI data. To clean data, we can use the remove component(s) option within the GIFT toolbox. ICA is a powerful method for cleaning fMRI data prior to dynamic and static FC studies, including for ROI-based analysis. In addition, we developed SimTB (A simulation toolbox for fMRI data) toolbox to generate simulate data and to test different analytical methods. SimTB gives users full control over generating data, including the creation of desirable spatial patterns of sources, the implementation of block- related and event-related experimental designs, the inclusion of tissue-specific baselines, and simulated head movement.

A video tutorial of the $\mathrm{dFC}$ pipeline is available at https://trendscenter.org/software/gift/videos. After adding the GIFT directory to the MATLAB search path, the GIFT toolbox can be launched by entering "gift" into the MATLAB command line. By clicking on the dynamic functional connectivity button, the "dynamic functional connectivity" toolbox appears and allows you to choose among several available dFC techniques (Figure 3). The $\mathrm{dFC}$ techniques are separated into two categories, temporal dynamics and spatial dynamics. 


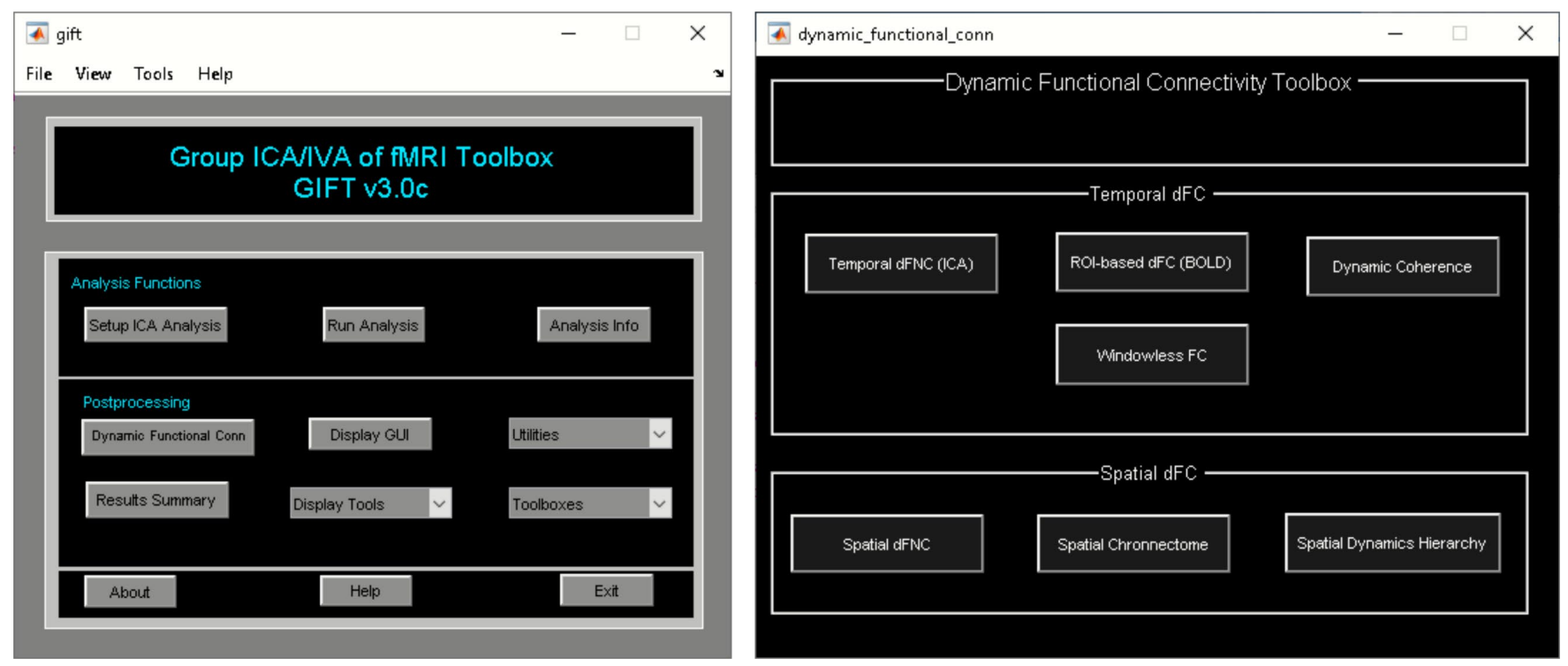

Figure 3. Left: GIFT Toolbox. Right: Dynamic Functional Connectivity Toolbox.

Temporal dynamic analyses ("Temporal $\mathrm{dFC}$ " options) study $\mathrm{dFC}$ via variations in the temporal patterns of sources, which are commonly achieved by studying the changes in their statistical dependency over time (Iraji et al., 2020). Spatial dynamic analyses ("Spatial dFC" options) focus on variations in the spatial patterns of sources over time (Iraji et al., 2020) (Figure 3). The list of measures available in GIFT to quantify dFC properties can be found in Table 1 .

Table 1. List of dynamic functional connectivity (dFC) measures.

For stateless dynamic measures:

- $\mathrm{dFC}$ variability: It represents the amount of variation in $\mathrm{dFC}$ over time and is commonly calculated as the standard deviation of $\mathrm{dFC}$ value across time/time windows.

- Coupling variability map: It is the spatial map of the amount of variation in the dFC of a given source/network over time, and it is estimated by calculating voxel-wise changes in the $\mathrm{dFC}$ of the source using the L1 norm distance (sum of absolute differences).

- Spatiotemporal transition matrix: It summarizes the whole brain $\mathrm{dFC}$ of a given source into a matrix in which each element of the matrix is the number of times that $\mathrm{dFC}$ value changes from one $\mathrm{FC}$ range to another. Several global metrics can be estimated from the spatiotemporal transition matrix including energy, entropy, and homogeneity.

For state-based dynamic measures:

- dFC strength: The strength of FC in a given state.

- Dwell time: The average amount of time that a subject lingers in each state. 
- Occupancy rate: The percentage of time that each state occurs during a scan.

- Transition matrix: The probability of transitioning from one state to another.

- Average variability index: It is an indication of the overall level of dynamism for a functional source. Variability index (VI) is defined as the standard deviation of the binomial distribution and estimates the level of variability in a region's association to a given source.

- Functional (inter-domain) state connectivity: It captures the level of concurrency between states of different sources (e.g., functional domains) when a technique (e.g., spatial dynamic hierarchy) estimates dynamic states for each source separately.

For meta-state dynamic measures:

- Number of realized meta-states: the number of distinct meta-states that an individual realizes during the length of a scan.

- Meta-state switching: the number of times an individual switches from one meta-state to a different metastate during the length of a scan.

- Meta-state span: Maximally different (in the L1 sense) meta-states that a given subject realizes.

- Meta-state total trajectory length: Total distance traveling in the state space which is the sum of L1 distances between successive meta-states for each subject.

- Level- $k$ hub meta-states: the meta-states that an individual visit at least $k$ times during the scan.

- Level-k transient meta-states: the meta-states that an individual visits less than $k$ times during the scan.

\section{Temporal dFC}

Temporal dFNC Toolbox: It is a WBA which computes whole-brain dFC (Allen et al., 2014). It uses ICA from the GIFT toolbox to estimate nodes/sources and their associated time course. To access temporal dFNC, click on Temporal dFNC (ICA) button under Temporal dFC options (Figure 3). Briefly, the steps include the following (Figure 4):

1) "Setup/Run Analysis" panel (Figure 5) in which we enter the TR of the experiment and organize components by functional domains/networks which will be useful in plotting FNC matrices at the end of the analysis. dFNC defaults menu allows users to choose different preprocessing options and $\mathrm{dFC}$ parameters. For preprocessing, options include detrending, despiking, low-pass/bandpass filtering, and regressing out confounding covariates from time courses. dFNC parameters include the parameters associated with window (e.g., window size, the alpha parameter of the Gaussian window) and the regularization method (L1 or none). After completing the parameter 
selection, use the Run button to run the dynamic FNC. Windowed dFNC matrices are saved for each subject.

2) "Post-processing" option (Figure 6) which consists of two panels: state-based dFNC and metastate dFNC (meta-state analysis). For state-based analysis, we can enter the number of k-means clusters (states) or estimate it using various algorithms, such as Gap statistic, AIC/BIC, Dunns Index, and Silhouette algorithms. We can also customize k-means options like the number of kmeans iterations, the distance metric, the maximum number of iterations, and the number of reference data-sets used for the gap statistic in "Cluster options" menu. For meta-state analysis, several methods like k-means, PCA, and various ICA techniques are available. It should be noted that "Post-processing" options are similar across different dFC analyses (some have already been implemented in GIFT); therefore, for the sake of brevity, we will not repeat them in the other techniques.

3) "Display" option allows us to visualize the result of both (meta-) state results, such as state dFNC, connectivity patterns of meta-state dFNC, and connectogrom plot (e.g., Figure 7).

4) "Stats" option provides various statistical analysis choices, like one sample t-test, two-sample ttest, and paired t-test on the dFNC strength of each state and (meta-) states metrics.

Temporal dFNC Toolbox also consists of "Task-based dFNC" option (Sakoglu et al., 2010), which uses experimental design information (regressors) as input. Regressors are obtained by convolving onsets with a hemodynamic response function (HRF). To compute task-based dFNC, click on the "Import Design" menu available in the "Setup/Run Analysis" panel (Figure 5). The sliding window approach is applied to the task-based regressors and ICA components' time courses. Correlation is computed between the windowed ICA time courses and windowed task-based regressors for each subject. Options are provided to apply statistical testing to the correlations. 


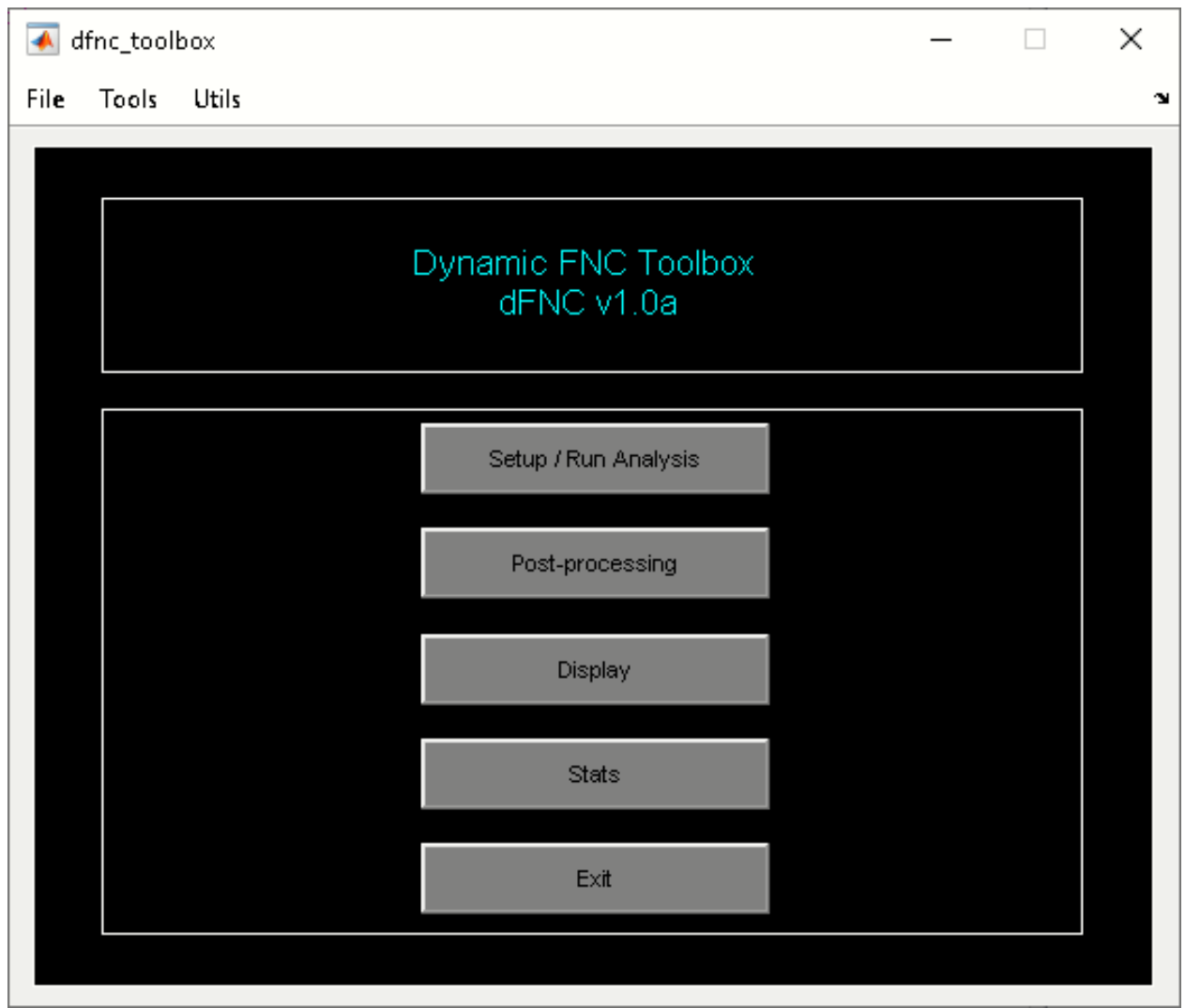

Figure 4. Temporal dFNC Toolbox

A Figure 1: dFNC Setup Analysis

dFNC-Defaults Import Design

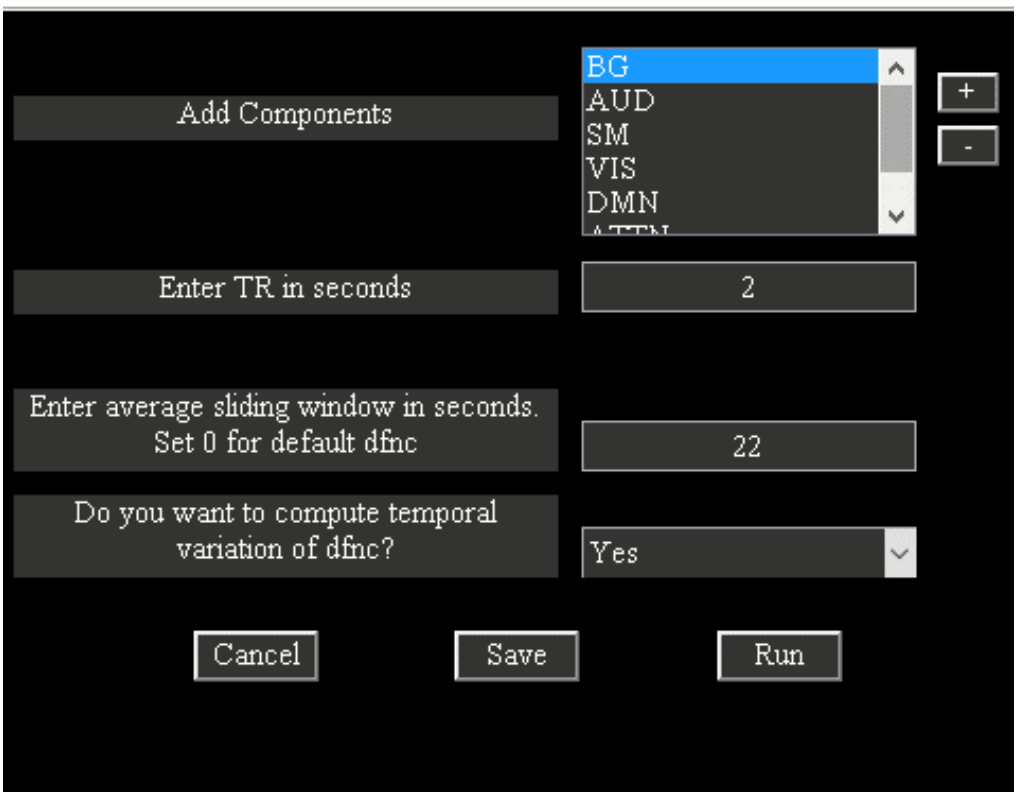

Figure 5. Temporal dFNC Setup Analysis 


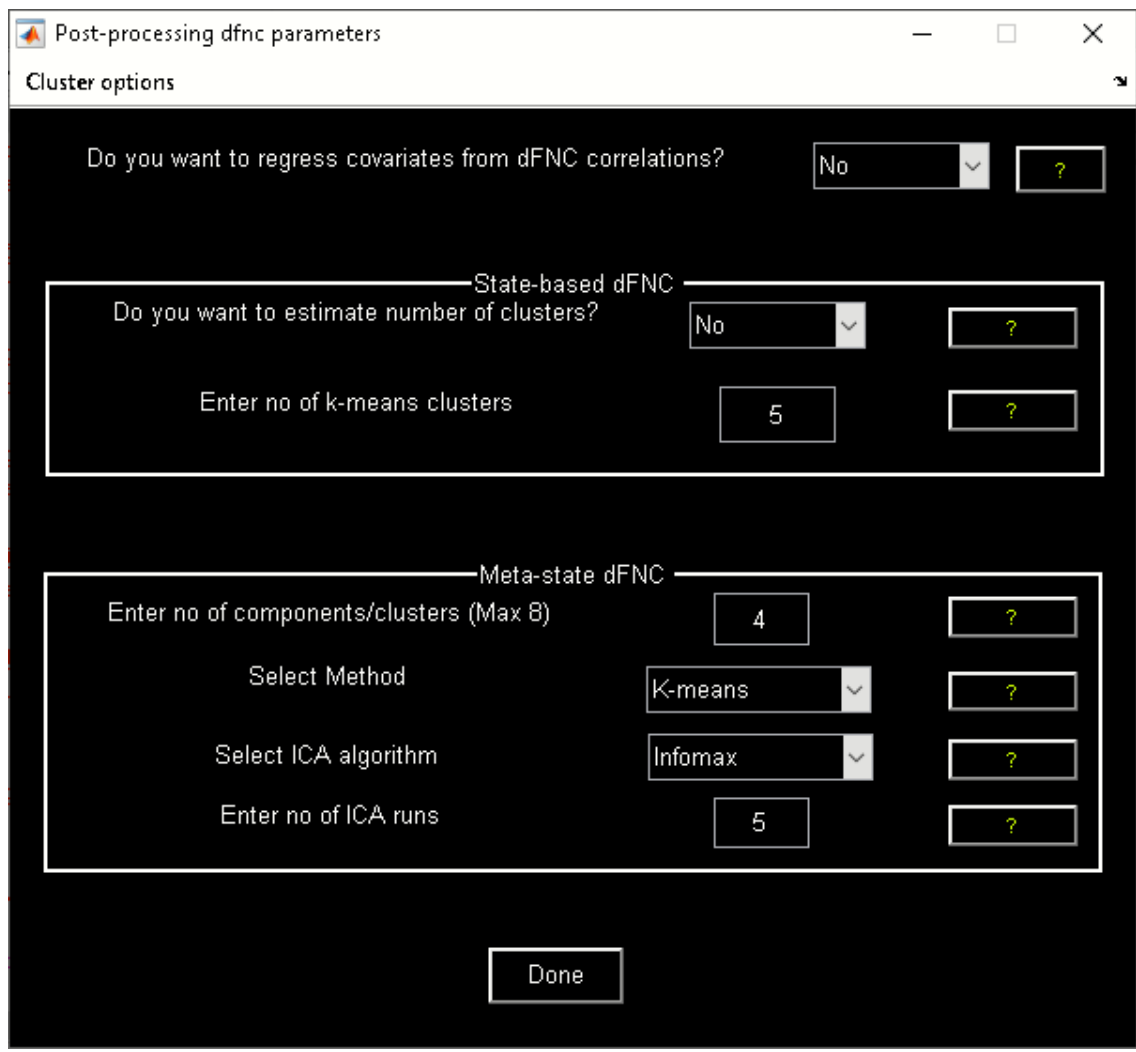

Figure 6. Post-processing dFNC

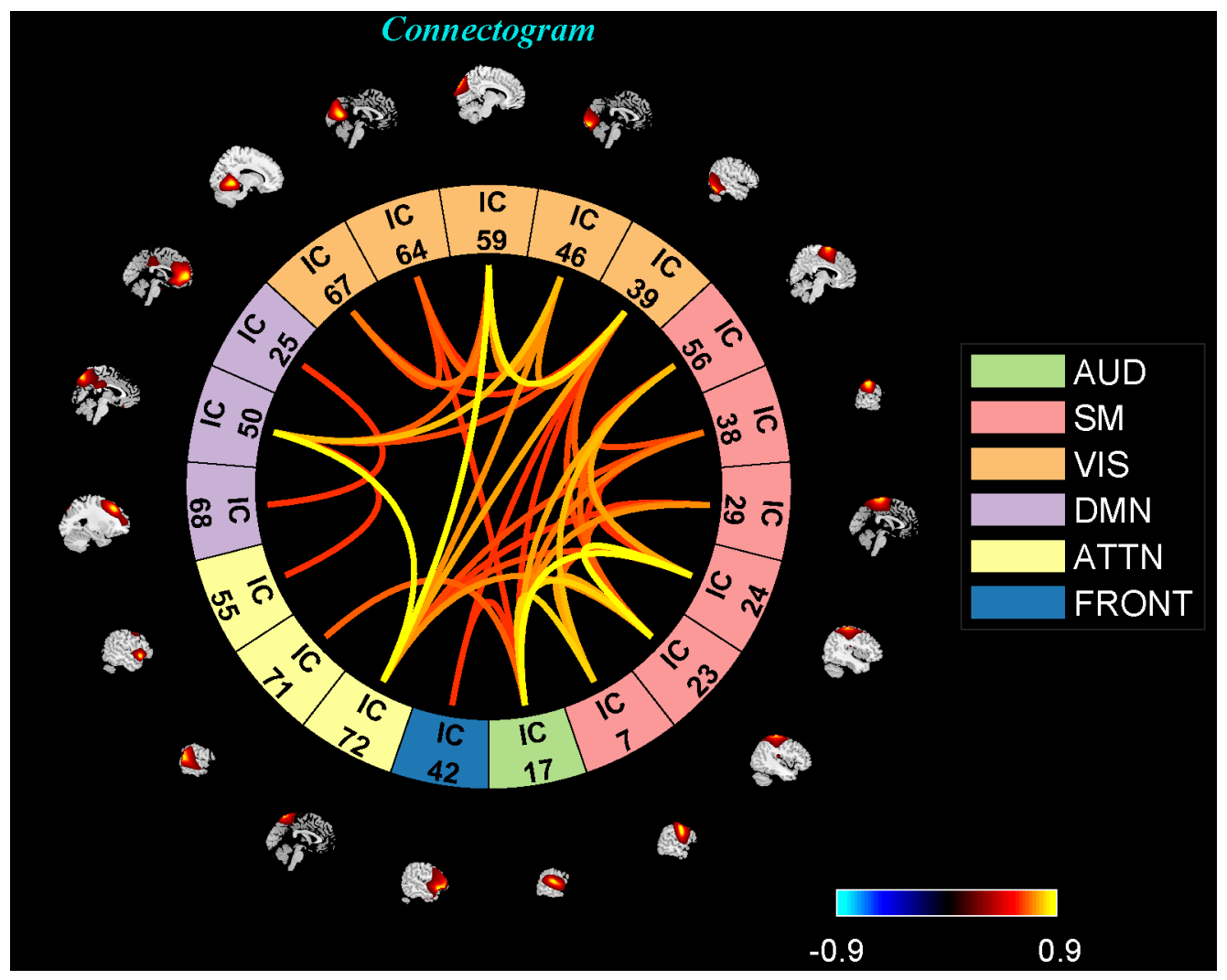

Figure 7. One of the Clusters represented as a Connectogram 
Temporal dFC (BOLD) Toolbox: In contrast to temporal dFNC, "Temporal dFC (BOLD)" uses predefined regions of interest (ROIs), and the average BOLD signals within ROIs are used to calculate dFC patterns at each window. It should be noted that we do not recommend using predefined ROIs to study $\mathrm{dFC}$ as they do not consider inter- and intra-subject variation. Two options are available in this toolbox. The first one is ROI-ROI dFC in which the user inputs ROIs mask, and WBA is used to estimate dFC between ROIs. The other option is to estimate ROI-to-voxel dFC in which windowed-FC is calculated between the average BOLD signal of each ROI and the BOLD signal of every voxel in the brain. After calculating windowed-FC, the rest of the analysis would be similar to temporal dFC analysis. For instance, k-mean clustering can be used to estimate dFC states, and we can perform meta-state analysis in the same manner.

Dynamic Coherence Toolbox: "Dynamic Coherence" applies complex Morlet wavelet on the time courses of ICNs to capture dFNC in the augmented time and frequency space (Yaesoubi et al., 2015a). In other words, it estimates dFC at different frequencies and phase lags. Dynamic Coherence Toolbox is divided into two parts (Figure 8):

1. "Setup/Run Analysis" allows us to enter analysis parameters. Options are provided to group components by network names and to enter experimental TR in seconds and complex k-means specific setting like the number of clusters and k-means replicates (Figure 9). There are options to preprocess the time courses like detrending, despiking, filtering, and regressing out variance associated with noise from the time courses when you click the "Dynamic Coherence Defaults" menu. After the analysis is complete, the cluster states information is saved to the disk.

2. "Display" option visualizes the results of the analysis, including estimated dFC states and associated frequency and phase histogram (e.g., Figure 10). 


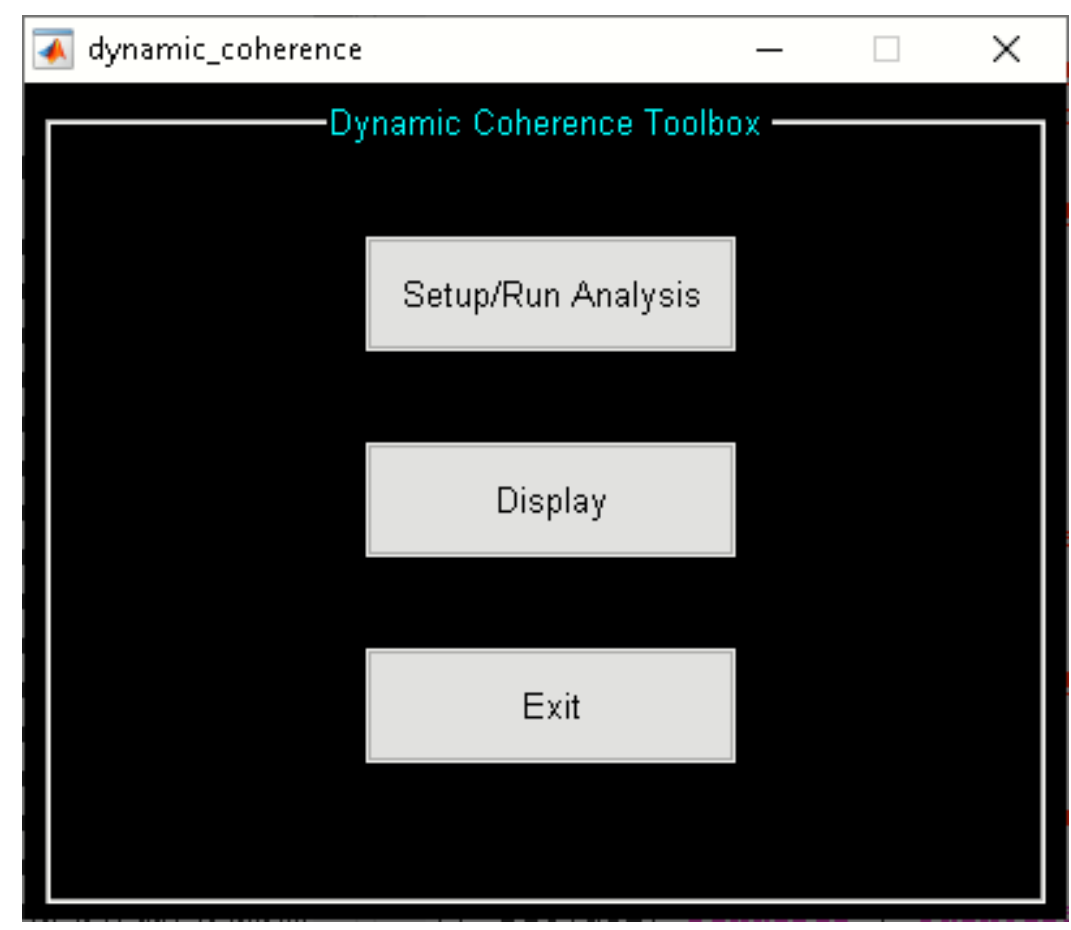

Figure 8. Dynamic Coherence Toolbox

Figure 1: Dynamic Coherence Setup Analysis

Dynamic Coherence Defaults

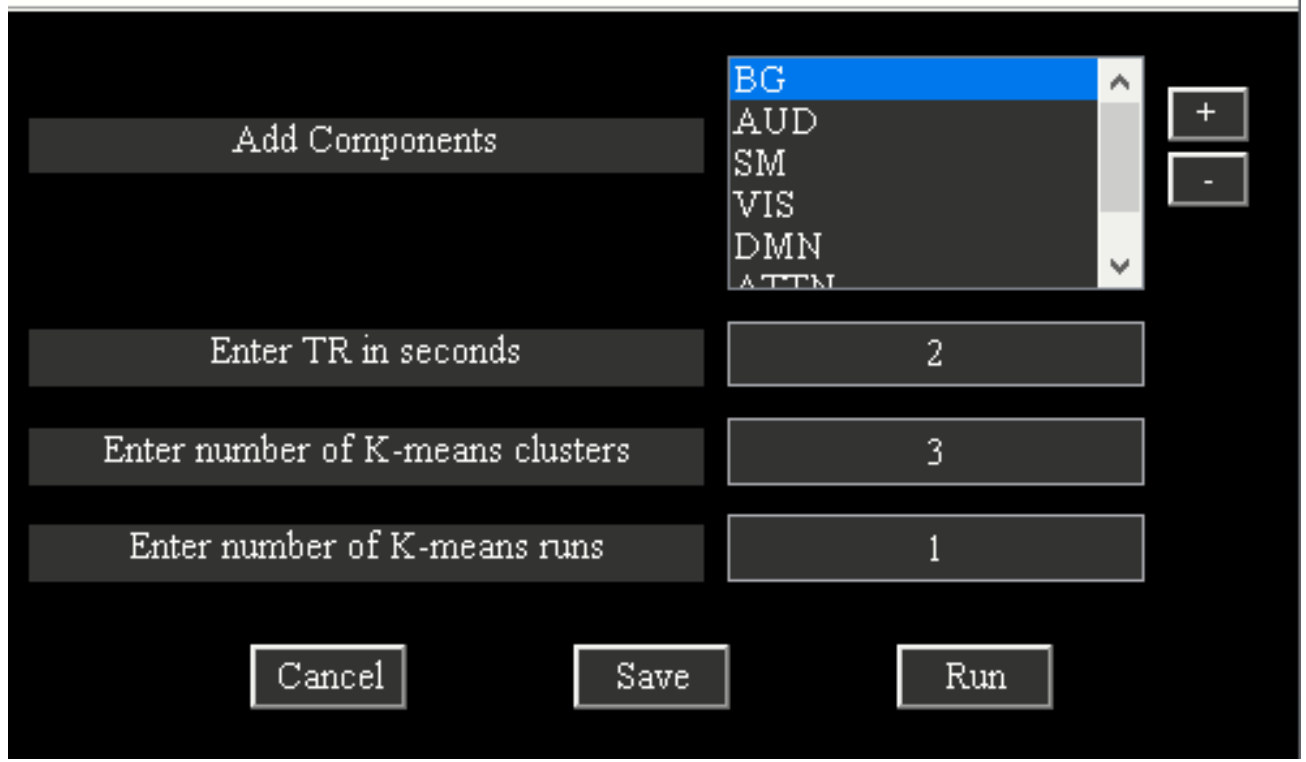

Figure 9. Dynamic Coherence Setup Analysis 

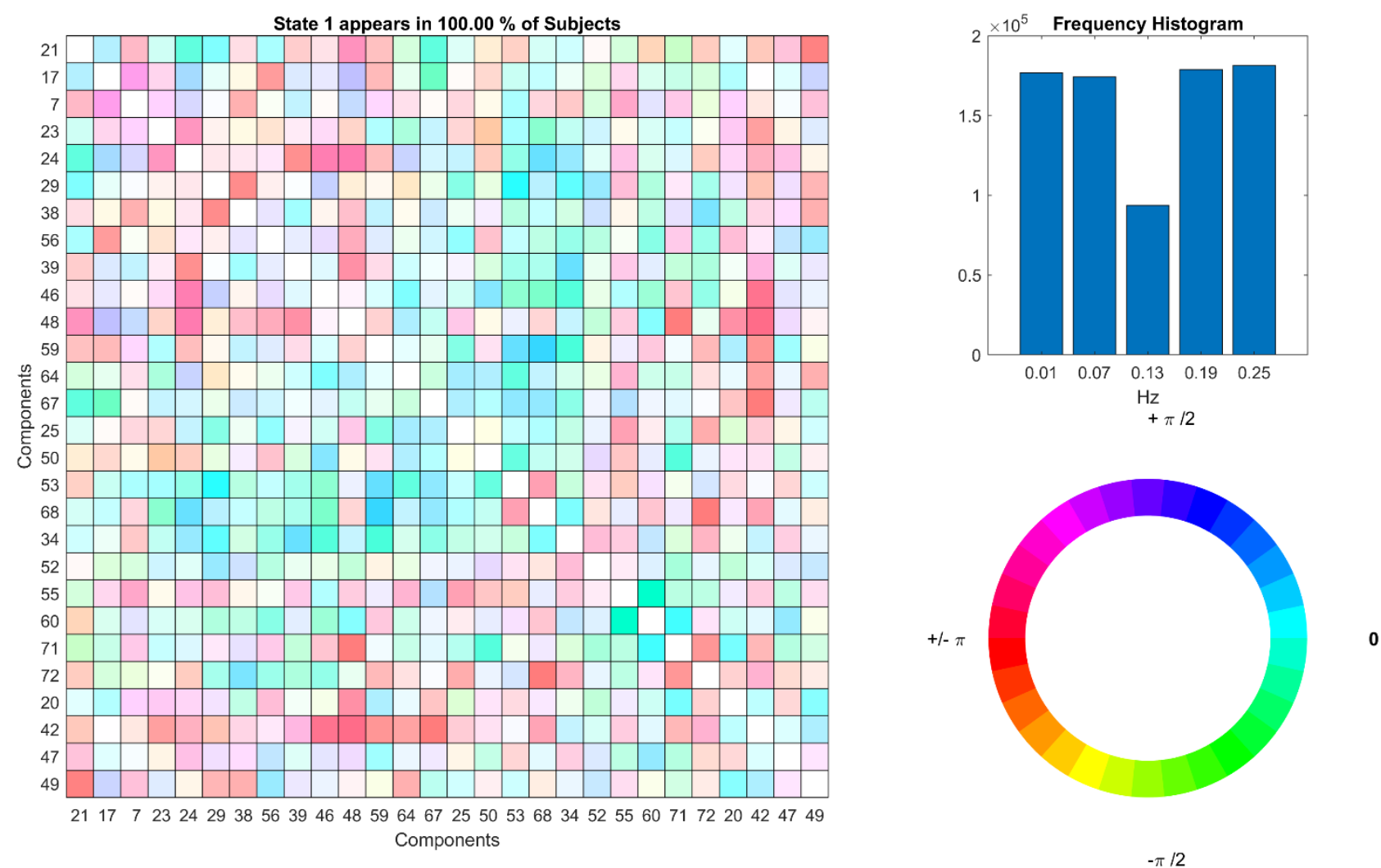

Figure 10. Dynamic Coherence Results

Windowless Functional Connectivity Toolbox: "Windowless FC" bypasses windowing operation by directly measuring linear dependence in the sample space (Yaesoubi et al., 2018). This approach calculates dFNC states as the outer product between the subspace bases estimated using K-SVD. As a result, it can detect $\mathrm{dFC}$ patterns with arbitrary rates of changes. The toolbox consists of two panels (Figure 11):

1. "Setup/Run Analysis" panel provides options for preprocessing the time courses of ICNs, selecting the number of dictionary elements, and the maximum number of iterations. After the analysis, dictionary elements and mixing coefficients are saved to the disk space.

2. "Display" panel allows users to organize components by networks for displaying purposes using matrix plots or a connectogram (e.g., Figure 12). 


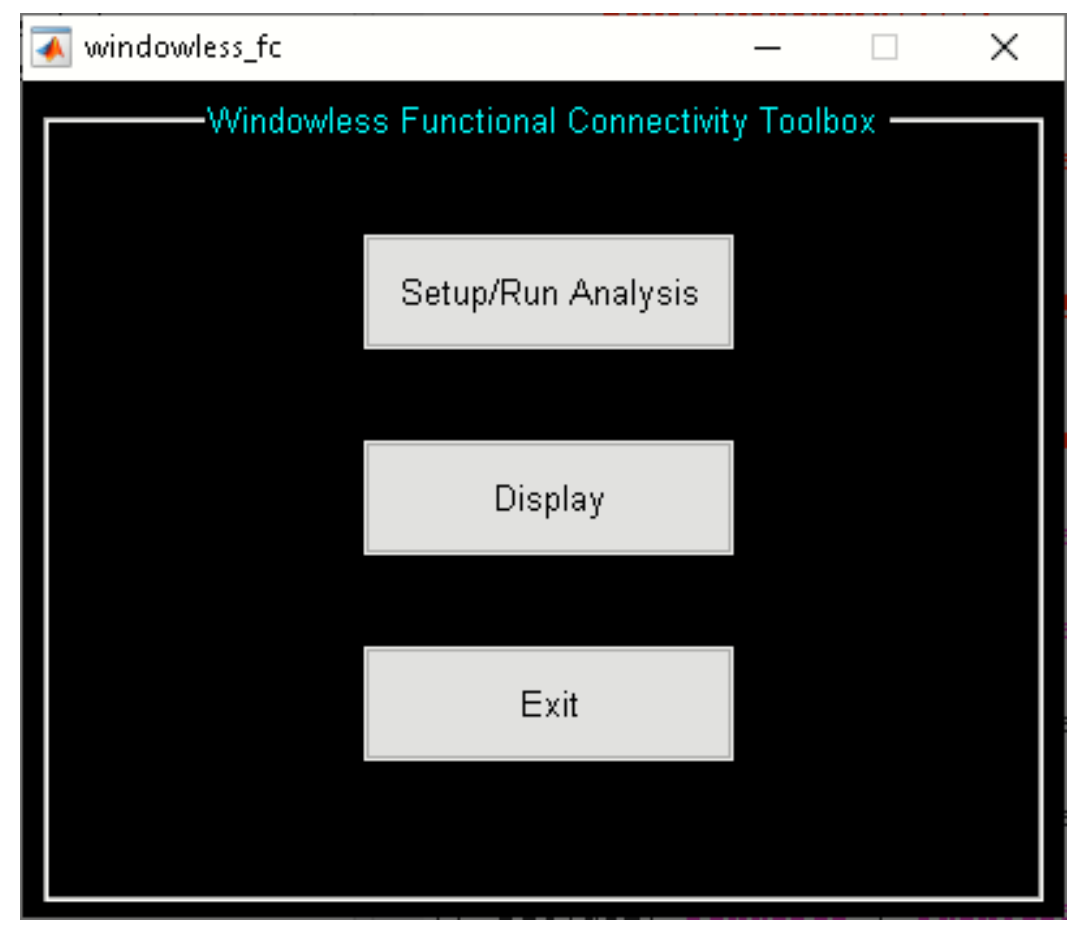

Figure 11. Windowless FC Toolbox

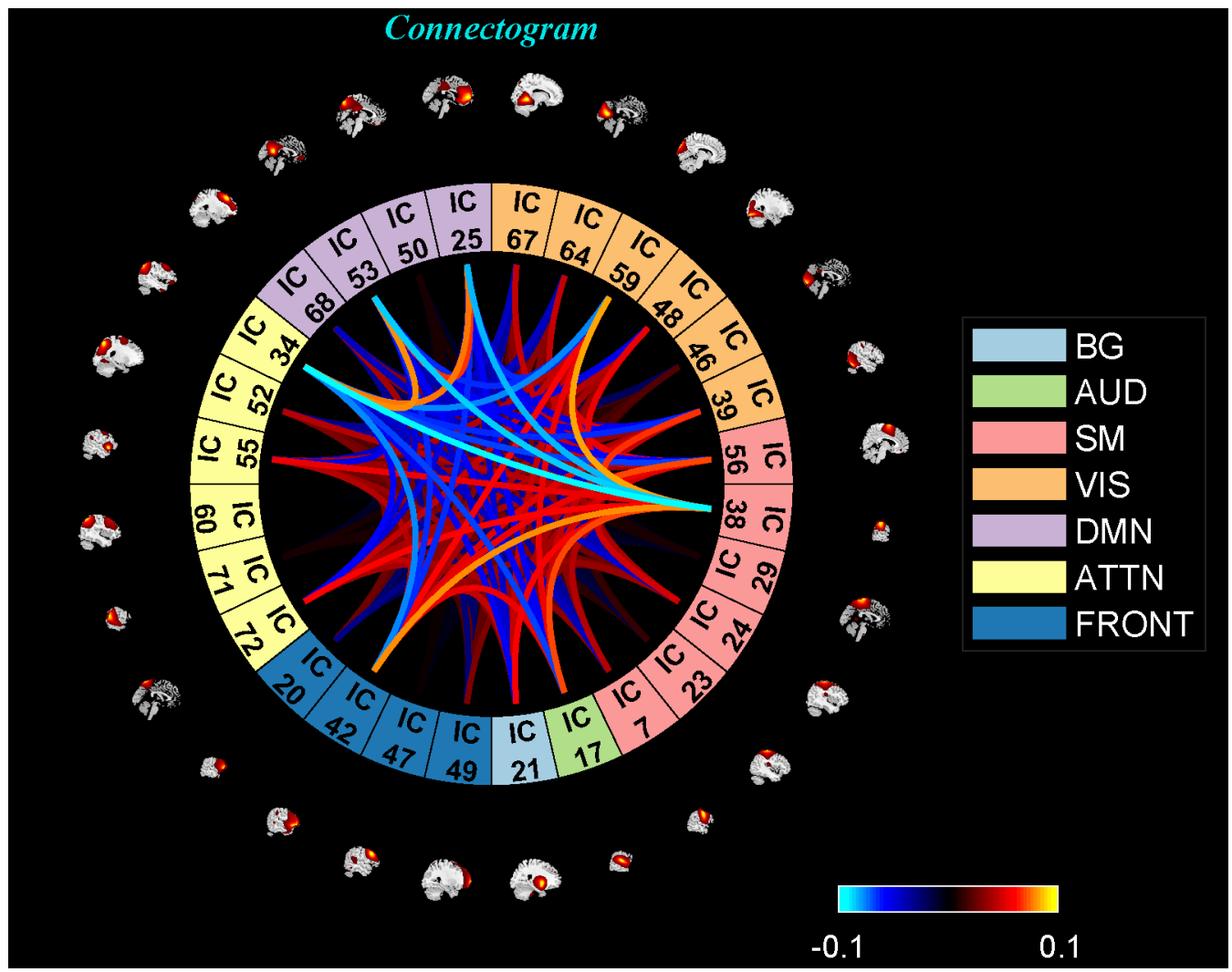

Figure 12. An example of one (K-SVD) dFC state 


\section{Spatial dFC}

Spatial dFNC Toolbox: "Spatial dFNC" treats data from each time window as a separate dataset for independent vector analysis (IVA) to capture variations in the spatial pattern of each source over time (Ma et al., 2014). The steps of spatial dFNC include (Figure 13):

1. "Setup/Run Analysis" in which we enter the window size, the number of IVA components, and the number of IVA run (Figure 14). We also assign subjects into different groups in this panel (Figure 14).

2. "Post-processing" in which we insert the parameters of Markov chain analysis and the threshold for t-tests (Figure 15).

3. "Display" in which all the spatial dFNC results (e.g., Figure 16) are summarized in an HTML page and shown in a web browser.

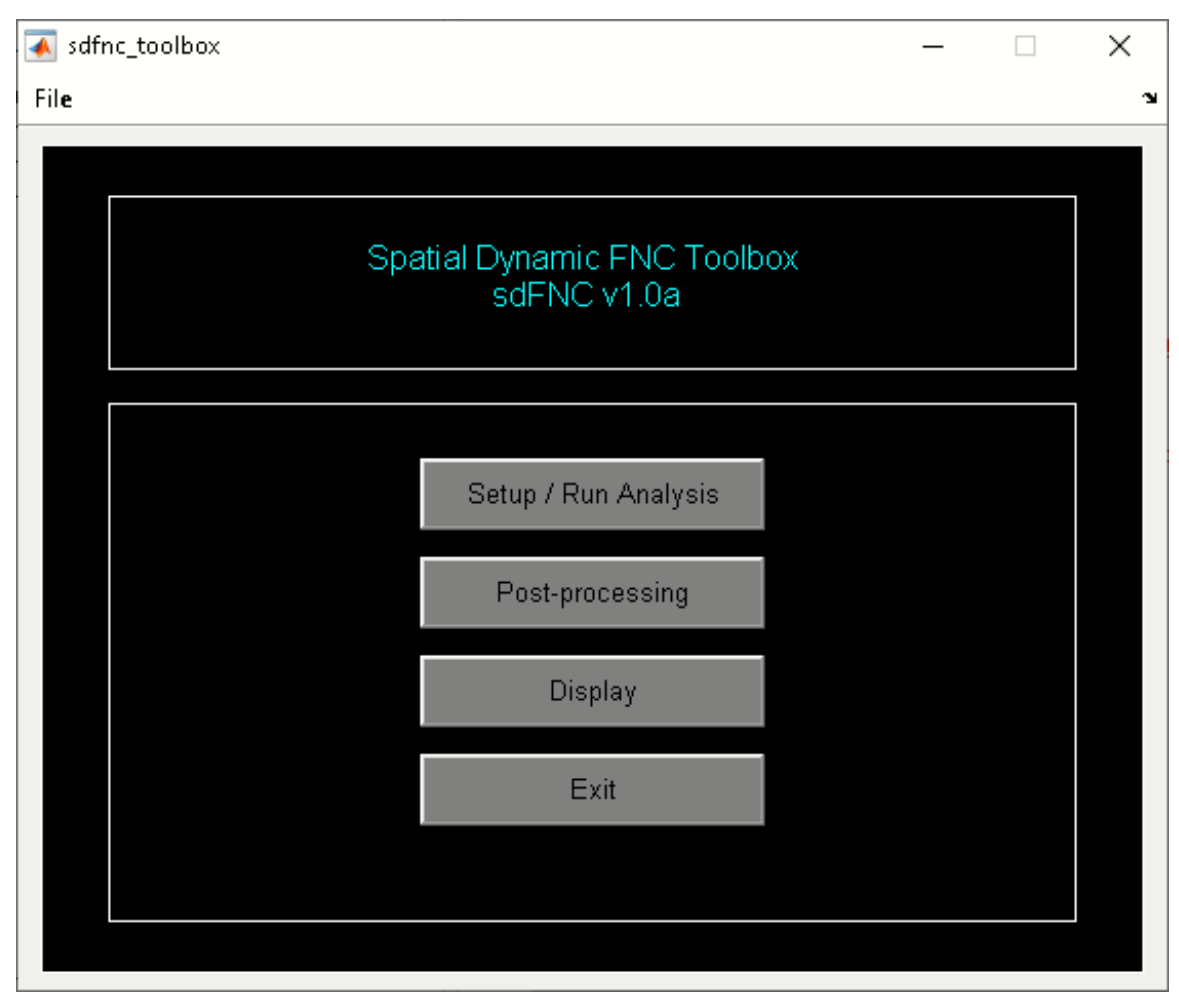

Figure 13. Spatial dFNC Toolbox 


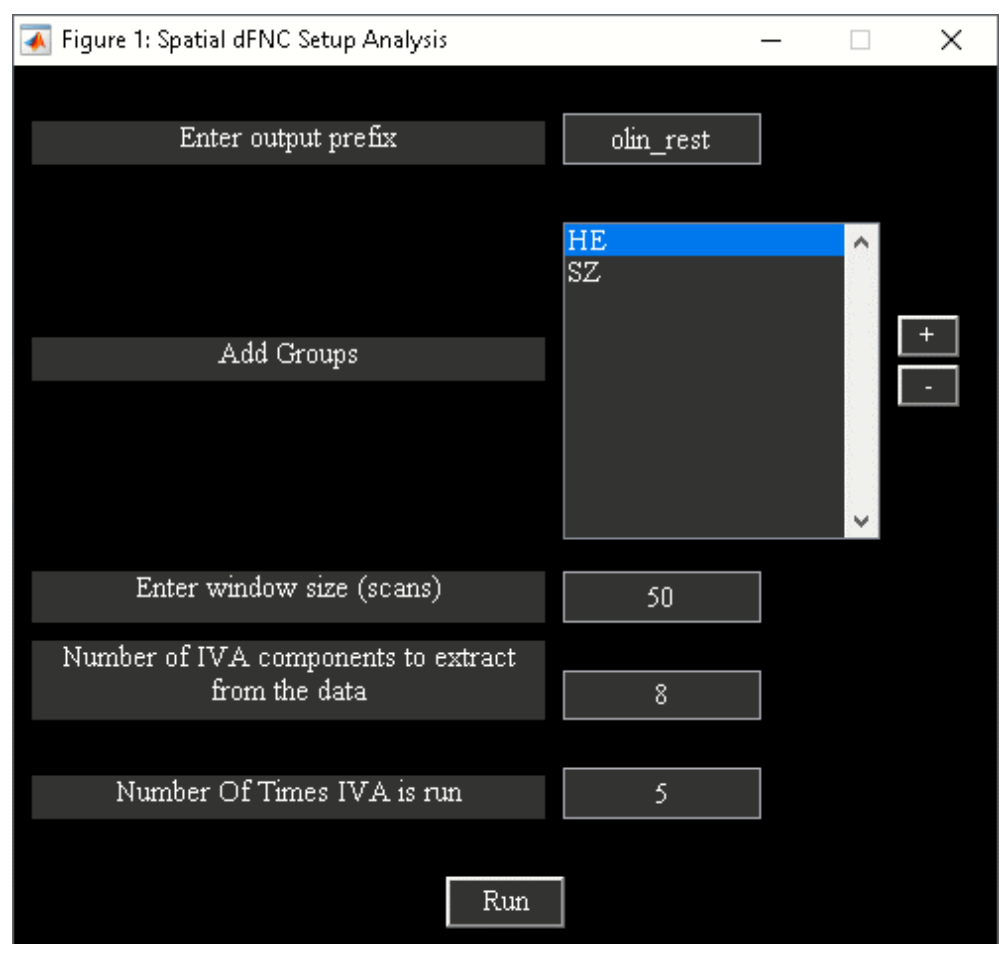

Figure 14. Spatial dFNC Setup Analysis

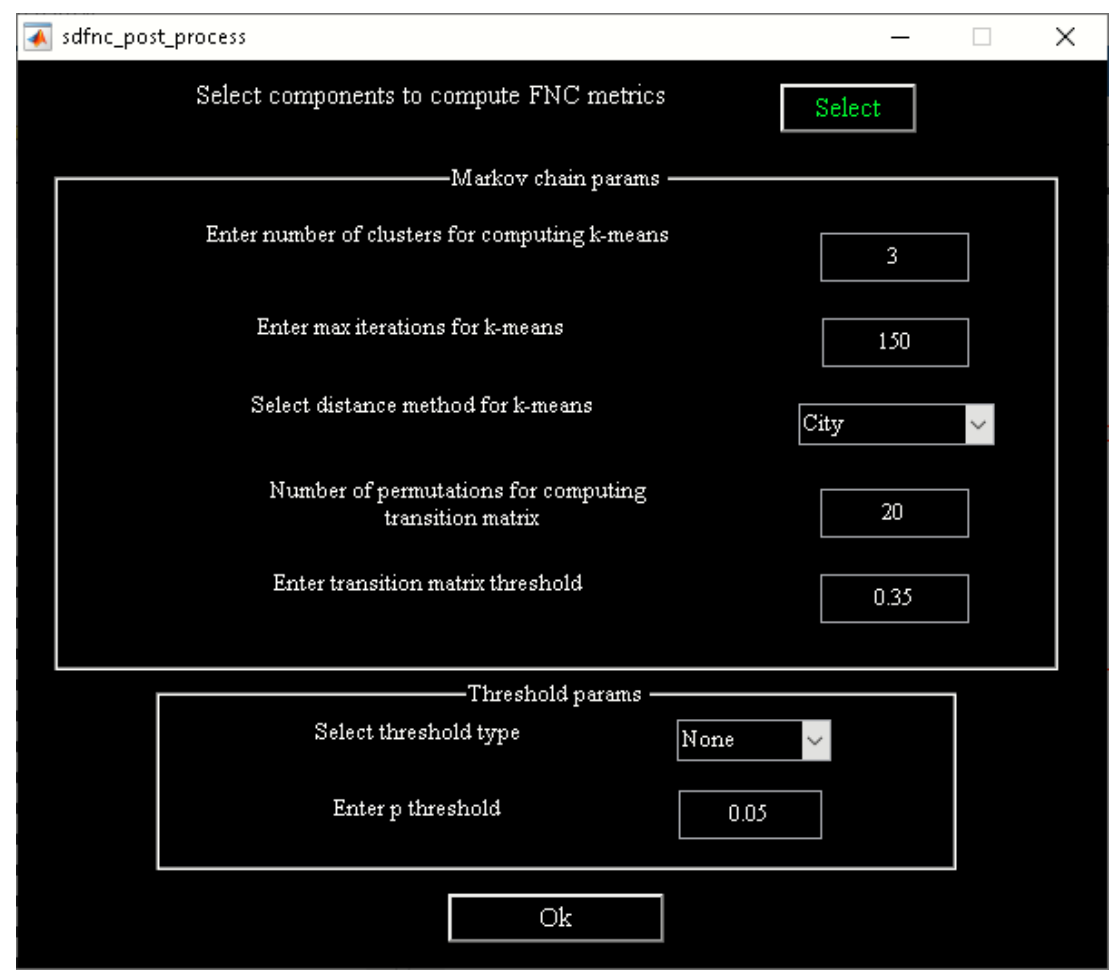

Figure 15. Spatial dFNC Post-processing GUI 


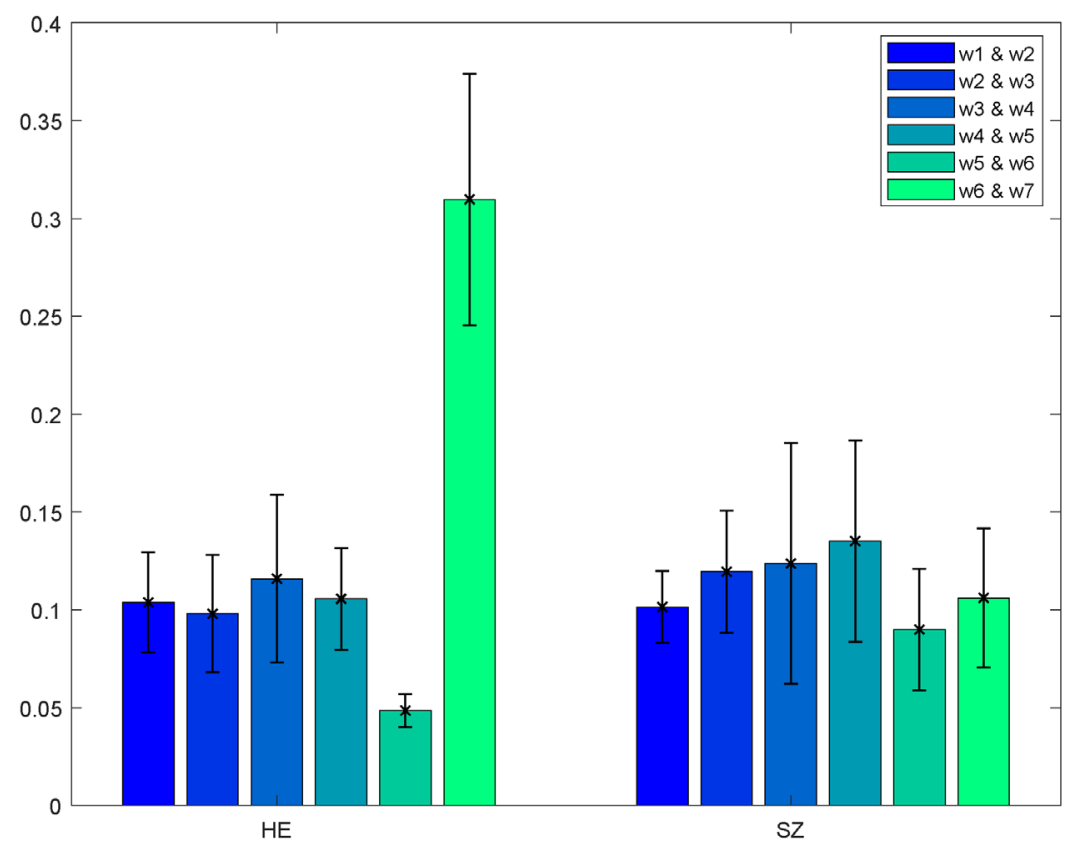

Figure 16. One example of the Spatial dFNC results. KL divergence is computed between pairs of windows.

Spatial Chronnectome Toolbox: "Spatial Chronnectome" captures on voxel-wise changes in the spatial patterns of sources over time. While the original work (Iraji et al., 2019a) used pair-wise correlation to calculate the association of each voxel to a given source/network regardless of its contribution to other sources, the partial correlation is also implemented in GIFT to evaluate the spatial dynamics of each source while controlling for the contribution of other sources. The toolbox uses ICA results from the GIFT toolbox to select the source of interest and their associated time courses.

The toolbox is divided into three steps (Figure 17):

1. "Setup/Run Analysis" in which we can enter the analysis parameters (Figure 18). When you click on Setup/Run, a figure window will open to select sources (ICA components) of interests and to enter experimental TR in seconds. "Spatial Chronnectome Defaults" menu contains options for preprocessing BOLD and ICA time courses as well as options for computing dynamic coupling maps in the "Dynamic Coupling Prefs" entry (Figure 19). BOLD signal and ICA components' time courses are preprocessed (despiking, filtering, and motion covariates variance removal) before calculating $\mathrm{dFC}$ maps. The parameters for sliding window procedures can be entered in "Dynamic Coupling Prefs."

2. "Post-processing" (Figure 20) in which we quantify the dFC properties. This step calculates the coupling variability map, spatiotemporal transition matrix, and spatial states associated with each 
source (network) and their properties such as dwell time, occupancy rate, and transition matrix. Parameters of k-means clustering like the number of clusters, the number of max iterations, and the distance metric can be selected here. We can also estimate the number of clusters using various algorithms, such as the gap statistic, Akaike/Bayesian information criteria (AIC/BIC), Dunns index, and Silhouette algorithms, available in the "cluster options" panel. A recent study compares a number of cluster validation indices (Vergara et al., 2020).

3. "Display" in which the results of spatial chronnectome analysis for each source of interest will be displayed (e.g., Figure 21).

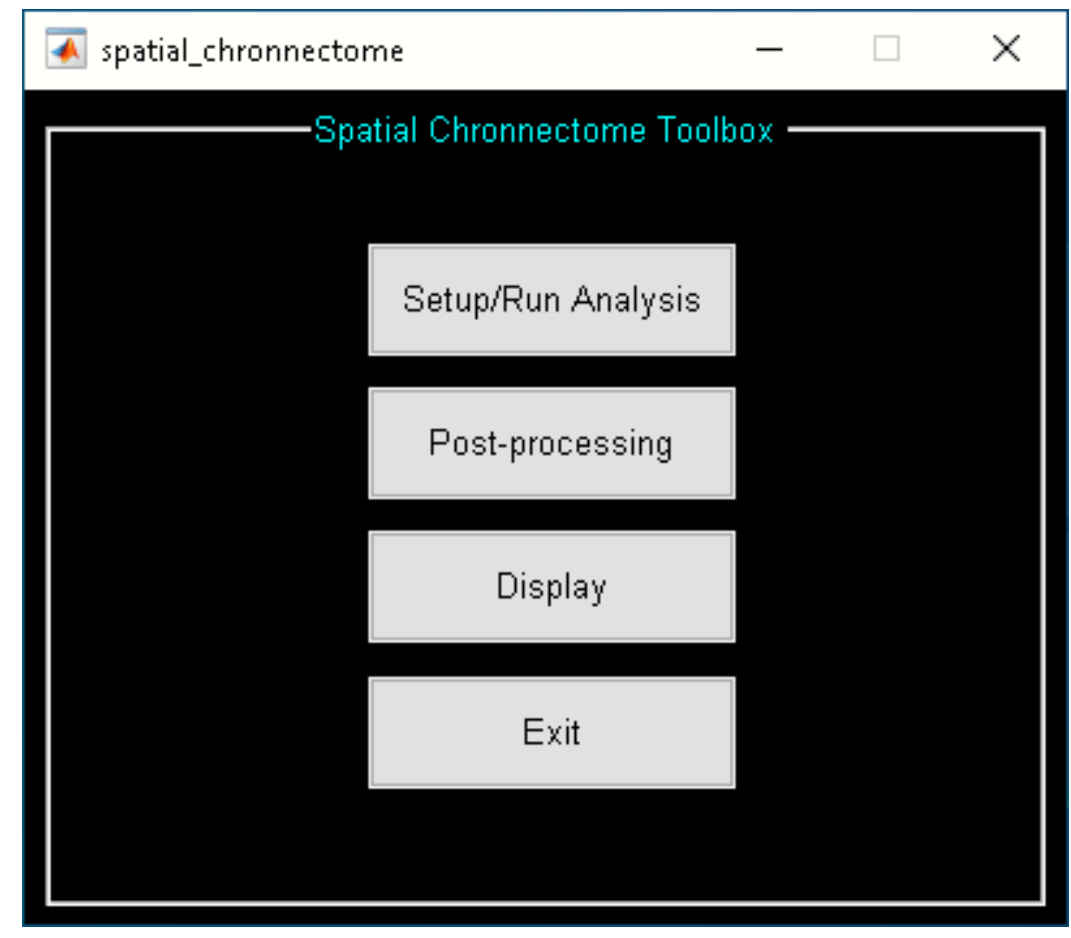

Figure 17. Spatial Chronnectome Toolbox 
Figure 1: Spatial Chronnectome Setup Analysis

SpatialChronnectome-Defaults

$\mathbf{3}$

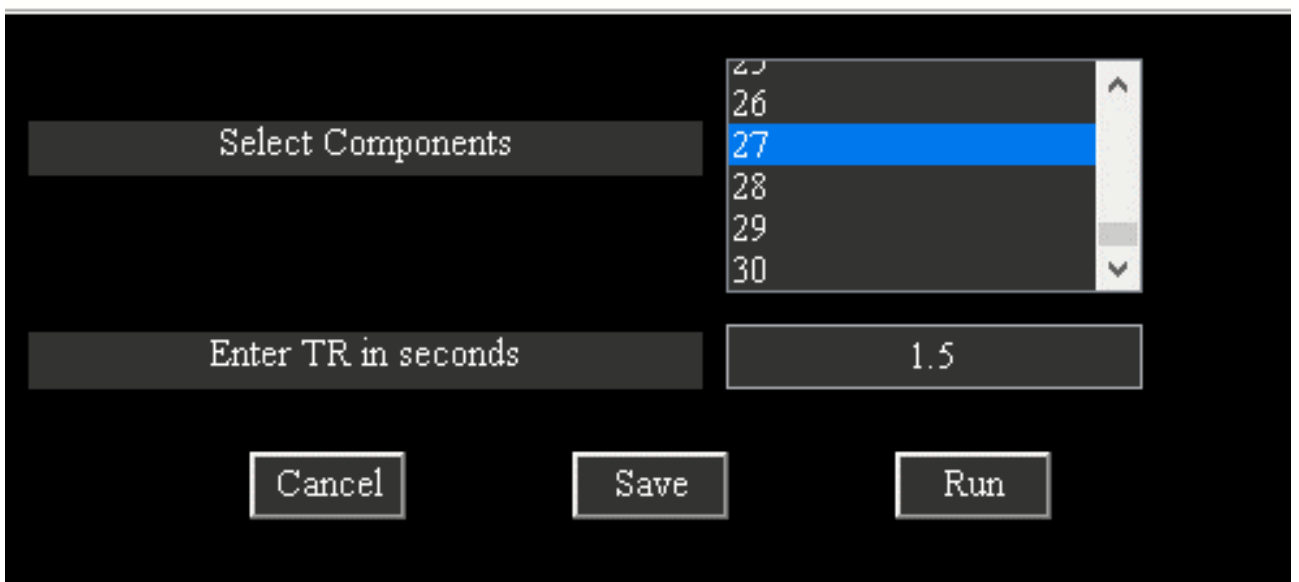

Figure 18. Spatial Chronnectome Setup Analysis
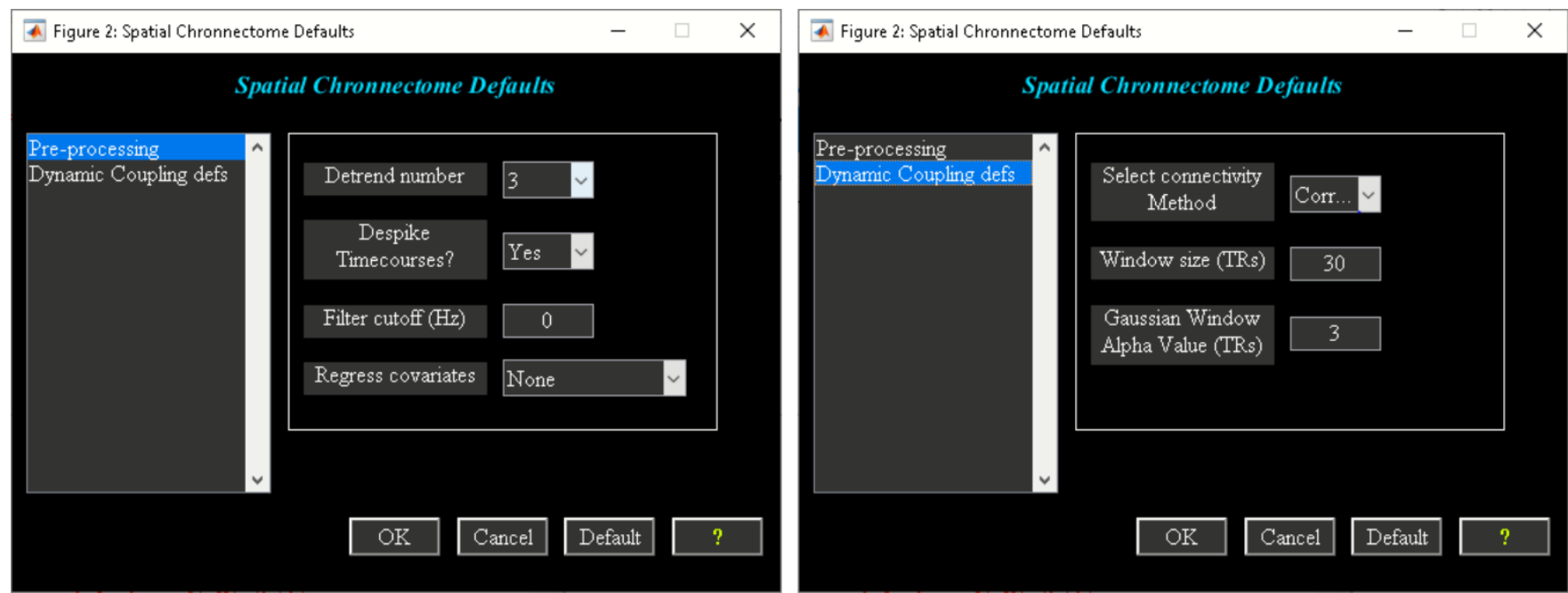

Figure 19. Spatial Chronnectome Defaults menu. Left: preprocessing options; Right: Dynamic Coupling Prefs options. 


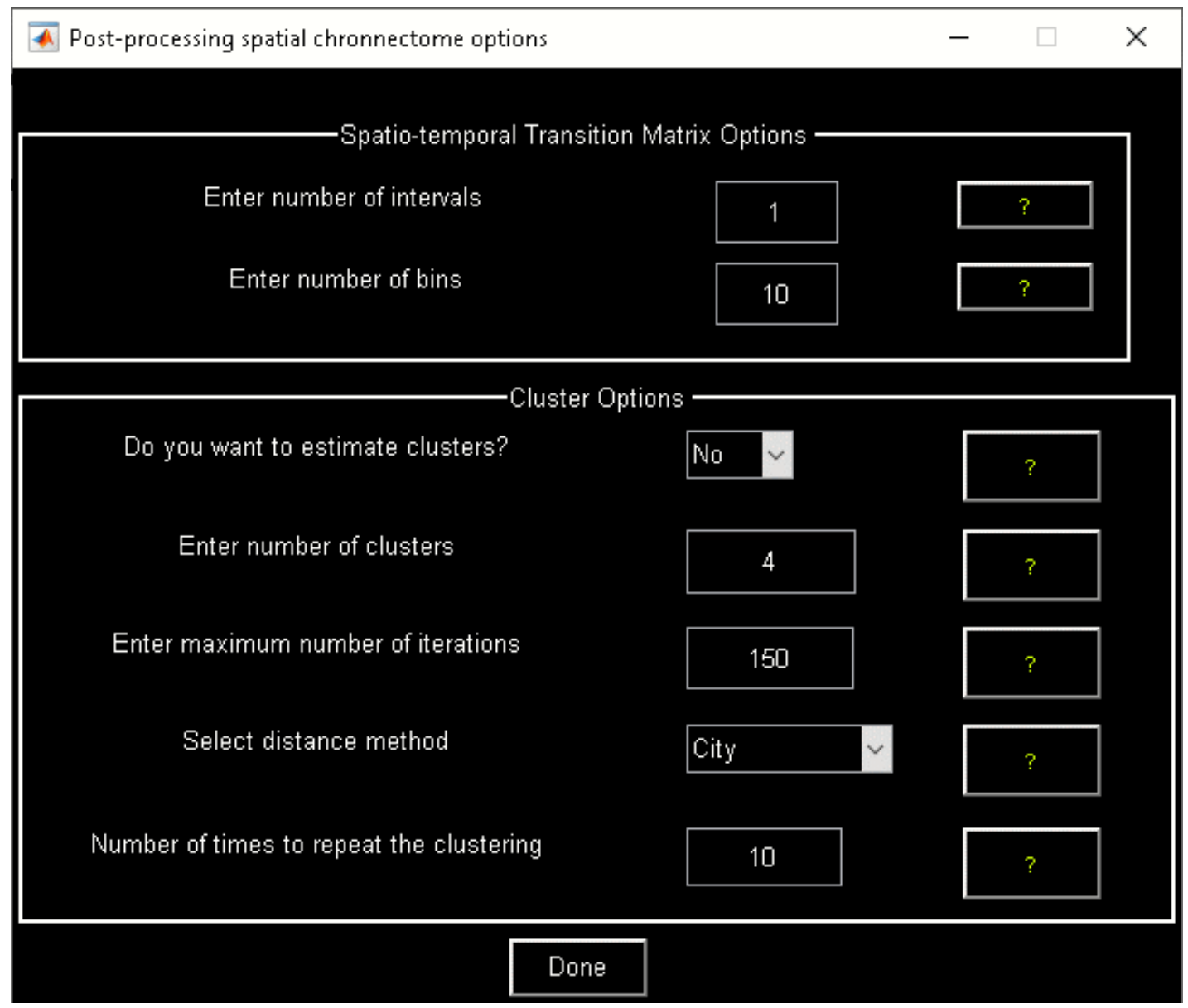

Figure 20. Spatial Chronnectome Post-processing.

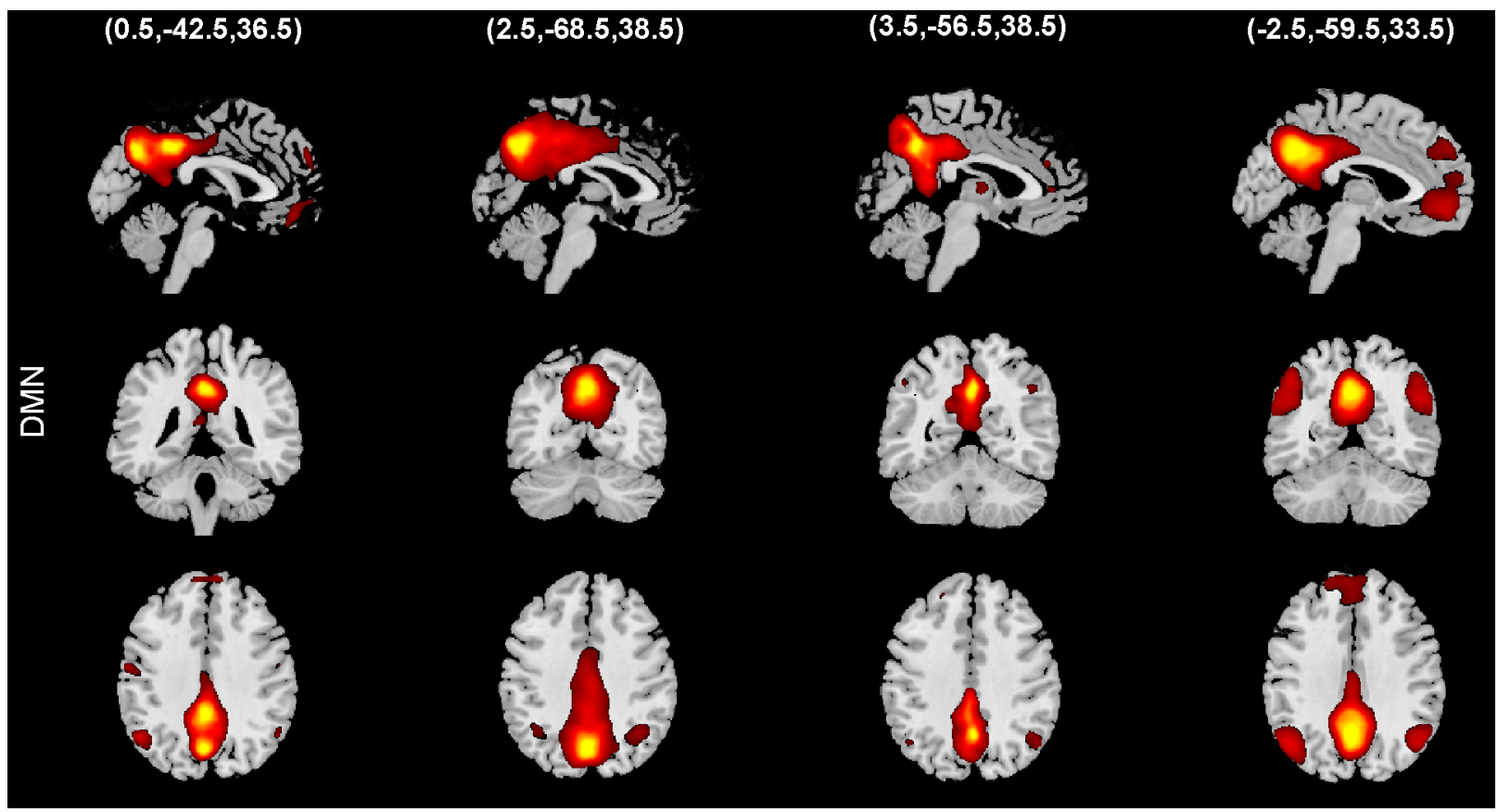

Figure 21. An example of Spatial Chronnectome Results. States are presented as vertically stacked orthogonal slices 
Spatial Dynamic Hierarchy Toolbox: The spatial dynamic hierarchy model studies the dynamic properties within brain hierarchy models. In the current version of GIFT (Version 4.0c), the method presented in (Iraji et al., 2019b) assumes fixed membership assignments between the elements of a hierarchy model and captures spatial dynamics within the functional domain as well as temporal dynamics within and between functional domains. Future versions will allow changes in membership assignments over time. This Toolbox is divided into three parts (Figure 22):

1. "Setup/Run Analysis" panel in which we enter the analysis parameters (Figure 23). We assign the components to functional domains and choose the parameters of clustering. Like other toolboxes, we can estimate the number of clusters using various algorithms, available in the "cluster options" menu. We can also choose parameters for preprocessing or cleaning ICA components' time courses, including despiking, low pass or bandpass filtering, and regressing out covariates.

2. "Post-processing" in which we quantify the dFC properties. This step calculates dFC states associated with each functional domain and their properties such as dwell time, occupancy rate, and transition matrix. It also computes Functional State Connectivity and associated Functional modules (FMs).

3. "Display" in which the results of spatial dynamic hierarchy analysis, such as states associated with each functional domain, are displayed (e.g., Figure 24).

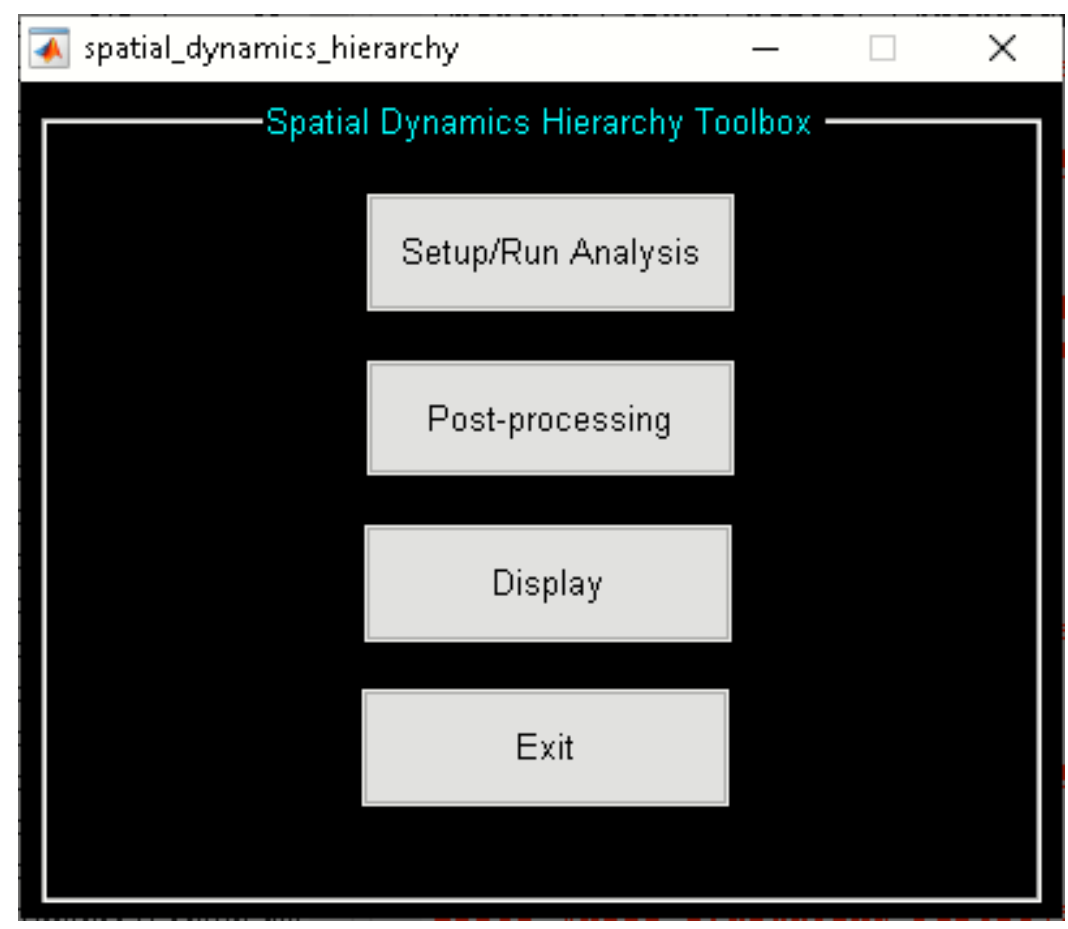

Figure 22. Spatial Dynamics Hierarchy Toolbox 


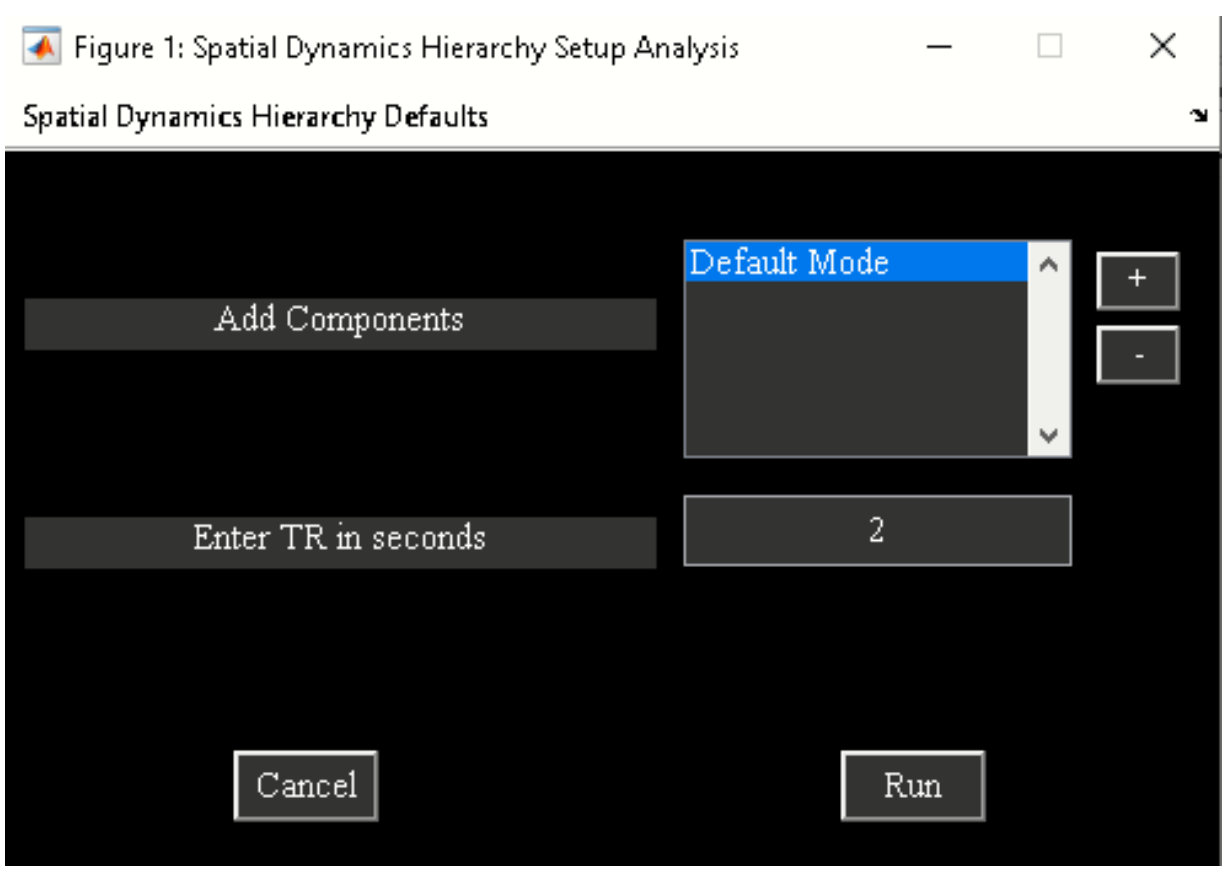

Figure 23. Spatial Dynamics Hierarchy Setup Analysis

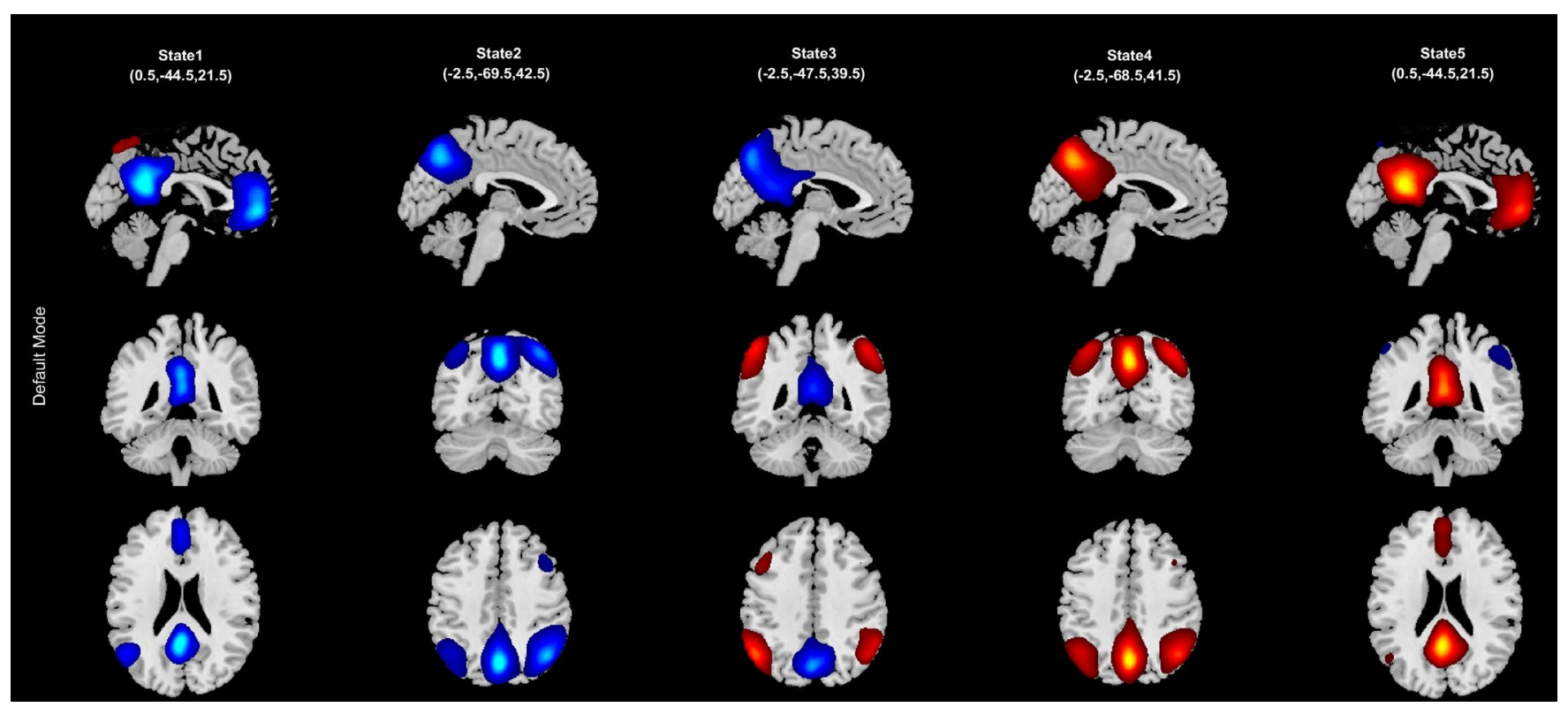

Figure 24. An example of spatial states for the default mode

It worth mentioning that we have developed other toolboxes to facilitate the advancement of neuroimaging research. For instance, the fusion ICA toolbox (FIT) contains several analytical techniques, such as joint ICA (Calhoun et al., 2006), parallel ICA (Liu et al., 2009) and CCA-Joint ICA (Sui et al., 2009), multiset canonical correlation analysis (MCCA) (Adali et al., 2015), transposed independent vector analysis (IVA) (Adali et al., 2015), Parallel-Group ICA + ICA (PGICA) (Qi et al., 2019), and deep fusion to analyze multimodal data (Plis et al., 2018). Joint ICA can be applied to different modalities (or task-fMRI) 
to extract maximally spatially independent maps for each modality (or task) that are coupled together by a shared loading parameter (in other words mixing coefficients is fixed between the modalities) (Calhoun et al., 2006). Parallel ICA is an extension of ICA that allows simultaneously running ICA on multiple modalities (Liu et al., 2009). For example, for two modalities, it extracts sources from both modalities and connections between them. Comparing with Joint ICA, where a shared mixing matrix is used for both modalities, parallel ICA assumes the two data sets are mixed in a similar pattern but not with identical parameters. CCA + joint ICA uses canonical correlation analysis (CCA) and ICA to extract both shared and distinct sources across features and mixing coefficients (Sui et al., 2009). MCCA estimates sources using the similarity in the mixing coefficients between the different modalities (Adali et al., 2015). Transposed IVA incorporates higher-order statistics in the MCCA model to extract common features across modalities (Adali et al., 2015). PGICA uses temporal information from first-level group ICA into a Parallel ICA framework. PGICA can detect linked FNC and structural covariations in the components when first-level fMRI and structural MRI (sMRI) data-sets are used (Qi et al., 2019). Deep Fusion uses neural networks for finding associations between $\mathrm{fMRI}$ and sMRI datasets (Plis et al., 2018). COINSTAC (Collaborative Informatics and Neuroimaging Suite Toolkit for Anonymous Computation) is another useful toolbox that was designed to address the need for sharing and collaboration in effective and easyto-use manner. COINSTAC provides tools to perform decentralized, privacy-enabled analysis to eliminate the need of directly sharing the data (Plis et al., 2016). We have implemented a number of algorithms within COINSTAC including preprocessing for fMRI, sMRI, diffusion MRI (dMRI) data as well as regression, group ICA, dynamic connectivity, support vector machine classification, and many more.

\section{Acknowledgment}

We are grateful for the opportunity to serve in this special issue on Tools of the Trade in SCAN. This work was supported by grants from the National Institutes of Health grant numbers 2R01EB005846, R01EB020407, R01MH118695, and P20GM103472; and National Science Foundation (NSF) grant 1539067 to Dr. Vince Calhoun.

\section{References}

Abrol, A., Damaraju, E., Miller, R. L., Stephen, J. M., Claus, E. D., Mayer, A. R. \& Calhoun, V. D. (2017) 'Replicability of time-varying connectivity patterns in large resting state fMRI samples', Neuroimage, 163, pp. 160-176. 
Adali, T., Levin-Schwartz, Y. \& Calhoun, V. D. (2015) 'Multi-modal data fusion using source separation: Two effective models based on ICA and IVA and their properties', Proc IEEE Inst Electr Electron Eng, 103(9), pp. 1478-1493.

Allen, E. A., Damaraju, E., Eichele, T., Wu, L. \& Calhoun, V. D. (2018) 'EEG Signatures of Dynamic Functional Network Connectivity States', Brain Topogr, 31(1), pp. 101-116.

Allen, E. A., Damaraju, E., Plis, S. M., Erhardt, E. B., Eichele, T. \& Calhoun, V. D. (2014) 'Tracking whole-brain connectivity dynamics in the resting state', Cereb Cortex, 24(3), pp. 663-676.

Allen, E. A., Erhardt, E. B., Damaraju, E., Gruner, W., Segall, J. M., Silva, R. F., Havlicek, M., Rachakonda, S., Fries, J., Kalyanam, R., Michael, A. M., Caprihan, A., Turner, J. A., Eichele, T., Adelsheim, S., Bryan, A. D., Bustillo, J., Clark, V. P., Feldstein Ewing, S. W., Filbey, F., Ford, C. C., Hutchison, K., Jung, R. E., Kiehl, K. A., Kodituwakku, P., Komesu, Y. M., Mayer, A. R., Pearlson, G. D., Phillips, J. P., Sadek, J. R., Stevens, M., Teuscher, U., Thoma, R. J. \& Calhoun, V. D. (2011) 'A baseline for the multivariate comparison of resting-state networks', Front Syst Neurosci, 5, p. 2.

Bassett, D. S., Porter, M. A., Wymbs, N. F., Grafton, S. T., Carlson, J. M. \& Mucha, P. J. (2013) 'Robust detection of dynamic community structure in networks', Chaos, 23(1), p. 013142.

Bressler, S. L. \& Menon, V. (2010) 'Large-scale brain networks in cognition: emerging methods and principles', Trends Cogn Sci, 14(6), pp. 277-290.

Caballero Gaudes, C., Petridou, N., Francis, S. T., Dryden, I. L. \& Gowland, P. A. (2013) 'Paradigm free mapping with sparse regression automatically detects single-trial functional magnetic resonance imaging blood oxygenation level dependent responses', Hum Brain Mapp, 34(3), pp. 501-518.

Cabral, J., Vidaurre, D., Marques, P., Magalhaes, R., Silva Moreira, P., Miguel Soares, J., Deco, G., Sousa, N. \& Kringelbach, M. L. (2017) 'Cognitive performance in healthy older adults relates to spontaneous switching between states of functional connectivity during rest', Sci Rep, 7(1), p. 5135.

Cai, B., Zille, P., Stephen, J. M., Wilson, T. W., Calhoun, V. D. \& Wang, Y. P. (2018) 'Estimation of Dynamic Sparse Connectivity Patterns From Resting State fMRI', IEEE Trans Med Imaging, 37(5), pp. 1224-1234.

Calhoun, V. D. \& Adali, T. (2012) 'Multisubject independent component analysis of fMRI: a decade of intrinsic networks, default mode, and neurodiagnostic discovery', IEEE Rev Biomed Eng, 5, pp. 60-73.

Calhoun, V. D. \& Adali, T. (2016) 'Time-Varying Brain Connectivity in fMRI Data: Whole-brain datadriven approaches for capturing and characterizing dynamic states', IEEE Signal Processing Magazine, 33(3), pp. 52-66.

Calhoun, V. D., Adali, T., Kiehl, K. A., Astur, R., Pekar, J. J. \& Pearlson, G. D. (2006) 'A method for multitask fMRI data fusion applied to schizophrenia', Hum Brain Mapp, 27(7), pp. 598-610.

Calhoun, V. D. \& de Lacy, N. (2017) 'Ten Key Observations on the Analysis of Resting-state Functional MR Imaging Data Using Independent Component Analysis', Neuroimaging Clin N Am, 27(4), pp. 561579 .

Calhoun, V. D., Miller, R., Pearlson, G. \& Adali, T. (2014) 'The chronnectome: time-varying connectivity networks as the next frontier in fMRI data discovery', Neuron, 84(2), pp. 262-274.

Chang, C. \& Glover, G. H. (2010) 'Time-frequency dynamics of resting-state brain connectivity measured with fMRI', Neuroimage, 50(1), pp. 81-98. 
Chang, C., Liu, Z., Chen, M. C., Liu, X. \& Duyn, J. H. (2013) 'EEG correlates of time-varying BOLD functional connectivity', Neuroimage, 72, pp. 227-236.

Chen, J., Sun, D., Shi, Y., Jin, W., Wang, Y., Xi, Q. \& Ren, C. (2018) 'Alterations of static functional connectivity and dynamic functional connectivity in motor execution regions after stroke', Neurosci Lett, 686, pp. 112-121.

Chen, T., Cai, W., Ryali, S., Supekar, K. \& Menon, V. (2016) 'Distinct Global Brain Dynamics and Spatiotemporal Organization of the Salience Network', PLoS Biol, 14(6), p. e1002469.

Chialvo, D. R. (2010) 'Emergent complex neural dynamics', Nature Physics, 6(10), pp. 744-750.

Choe, A. S., Nebel, M. B., Barber, A. D., Cohen, J. R., Xu, Y., Pekar, J. J., Caffo, B. \& Lindquist, M. A. (2017) 'Comparing test-retest reliability of dynamic functional connectivity methods', Neuroimage, 158, pp. $155-175$.

Cohen, J. R. (2018) 'The behavioral and cognitive relevance of time-varying, dynamic changes in functional connectivity', Neuroimage, 180(Pt B), pp. 515-525.

Cordova-Palomera, A., Kaufmann, T., Persson, K., Alnaes, D., Doan, N. T., Moberget, T., Lund, M. J., Barca, M. L., Engvig, A., Braekhus, A., Engedal, K., Andreassen, O. A., Selbaek, G. \& Westlye, L. T. (2017) 'Disrupted global metastability and static and dynamic brain connectivity across individuals in the Alzheimer's disease continuum', Sci Rep, 7, p. 40268.

d'Ambrosio, A., Valsasina, P., Gallo, A., De Stefano, N., Pareto, D., Barkhof, F., Ciccarelli, O., Enzinger, C., Tedeschi, G., Stromillo, M. L., Arevalo, M. J., Hulst, H. E., Muhlert, N., Koini, M., Filippi, M. \& Rocca, M. A. (2019) 'Reduced dynamics of functional connectivity and cognitive impairment in multiple sclerosis', Mult Scler, p. 1352458519837707.

Damaraju, E., Allen, E. A., Belger, A., Ford, J. M., McEwen, S., Mathalon, D. H., Mueller, B. A., Pearlson, G. D., Potkin, S. G., Preda, A., Turner, J. A., Vaidya, J. G., van Erp, T. G. \& Calhoun, V. D. (2014) 'Dynamic functional connectivity analysis reveals transient states of dysconnectivity in schizophrenia', Neuroimage Clin, 5, pp. 298-308.

de Lacy, N. \& Calhoun, V. D. (2019) 'Dynamic connectivity and the effects of maturation in youth with attention deficit hyperactivity disorder', Netw Neurosci, 3(1), pp. 195-216.

de Lacy, N., Doherty, D., King, B. H., Rachakonda, S. \& Calhoun, V. D. (2017) 'Disruption to control network function correlates with altered dynamic connectivity in the wider autism spectrum', Neuroimage Clin, 15, pp. 513-524.

Demirtas, M., Tornador, C., Falcon, C., Lopez-Sola, M., Hernandez-Ribas, R., Pujol, J., Menchon, J. M., Ritter, P., Cardoner, N., Soriano-Mas, C. \& Deco, G. (2016) 'Dynamic functional connectivity reveals altered variability in functional connectivity among patients with major depressive disorder', Hum Brain Mapp, 37(8), pp. 2918-2930.

Díez-Cirarda, M., Strafella, A. P., Kim, J., Peña, J., Ojeda, N., Cabrera-Zubizarreta, A. \& Ibarretxe-Bilbao, N. (2018) 'Dynamic functional connectivity in Parkinson's disease patients with mild cognitive impairment and normal cognition', Neuroimage Clin, 17, pp. 847-855.

Douw, L., Leveroni, C. L., Tanaka, N., Emerton, B. C., Cole, A. J., Reinsberger, C. \& Stufflebeam, S. M. (2015) 'Loss of resting-state posterior cingulate flexibility is associated with memory disturbance in left temporal lobe epilepsy', PLoS One, 10(6), p. e0131209. 
Douw, L., Wakeman, D. G., Tanaka, N., Liu, H. \& Stufflebeam, S. M. (2016) 'State-dependent variability of dynamic functional connectivity between frontoparietal and default networks relates to cognitive flexibility', Neuroscience, 339, pp. 12-21.

Eavani, H., Satterthwaite, T. D., Gur, R. E., Gur, R. C. \& Davatzikos, C. (2013) 'Unsupervised learning of functional network dynamics in resting state fMRI', Inf Process Med Imaging, 23, pp. 426-437.

Elton, A. \& Gao, W. (2015) 'Task-related modulation of functional connectivity variability and its behavioral correlations', Hum Brain Mapp, 36(8), pp. 3260-3272.

Engels, G., Vlaar, A., McCoy, B., Scherder, E. \& Douw, L. (2018) 'Dynamic Functional Connectivity and Symptoms of Parkinson's Disease: A Resting-State fMRI Study', Front Aging Neurosci, 10, p. 388.

Faghiri, A., Iraji, A., Damaraju, E., Belger, A., Ford, J., Mathalon, D., McEwen, S., Mueller, B., Pearlson, G., Preda, A., Turner, J., Vaidya, J. G., Van Erp, T. \& Calhoun, V. D. (2020) 'Weighted average of shared trajectory: A new estimator for dynamic functional connectivity efficiently estimates both rapid and slow changes over time', J Neurosci Methods, 334, p. 108600.

Faghiri, A., Iraji, A., Damaraju, E., Turner, J. \& Calhoun, V. D. (2019) 'Connectivity domain analysis: characterization of connectivity frequency profile using filter banks', bioRxiv, p. 706838.

Fu, Z., Caprihan, A., Chen, J., Du, Y., Adair, J. C., Sui, J., Rosenberg, G. A. \& Calhoun, V. D. (2019a) 'Altered static and dynamic functional network connectivity in Alzheimer's disease and subcortical ischemic vascular disease: shared and specific brain connectivity abnormalities', Hum Brain Mapp, 40(11), pp. 3203-3221.

Fu, Z., Chan, S. C., Di, X., Biswal, B. \& Zhang, Z. (2014) 'Adaptive covariance estimation of nonstationary processes and its application to infer dynamic connectivity from fMRI', IEEE Trans Biomed Circuits Syst, 8(2), pp. 228-239.

Fu, Z., Tu, Y., Di, X., Du, Y., Pearlson, G. D., Turner, J. A., Biswal, B. B., Zhang, Z. \& Calhoun, V. D. (2018) 'Characterizing dynamic amplitude of low-frequency fluctuation and its relationship with dynamic functional connectivity: An application to schizophrenia', Neuroimage, 180(Pt B), pp. 619-631.

Fu, Z., Tu, Y., Di, X., Du, Y., Sui, J., Biswal, B. B., Zhang, Z., de Lacy, N. \& Calhoun, V. D. (2019b) 'Transient increased thalamic-sensory connectivity and decreased whole-brain dynamism in autism', Neuroimage, 190, pp. 191-204.

Gitelman, D. R., Penny, W. D., Ashburner, J. \& Friston, K. J. (2003) 'Modeling regional and psychophysiologic interactions in fMRI: the importance of hemodynamic deconvolution', Neuroimage, 19(1), pp. 200-207.

Gonzalez-Castillo, J., Hoy, C. W., Handwerker, D. A., Robinson, M. E., Buchanan, L. C., Saad, Z. S. \& Bandettini, P. A. (2015) 'Tracking ongoing cognition in individuals using brief, whole-brain functional connectivity patterns', Proc Natl Acad Sci US A, 112(28), pp. 8762-8767.

Guo, X., Duan, X., Suckling, J., Chen, H., Liao, W., Cui, Q. \& Chen, H. (2019) 'Partially impaired functional connectivity states between right anterior insula and default mode network in autism spectrum disorder', Hum Brain Mapp, 40(4), pp. 1264-1275.

Hahamy, A., Calhoun, V., Pearlson, G., Harel, M., Stern, N., Attar, F., Malach, R. \& Salomon, R. (2014) 'Save the global: global signal connectivity as a tool for studying clinical populations with functional magnetic resonance imaging', Brain Connect, 4(6), pp. 395-403. 
Harlalka, V., Bapi, R. S., Vinod, P. K. \& Roy, D. (2019) 'Atypical Flexibility in Dynamic Functional Connectivity Quantifies the Severity in Autism Spectrum Disorder', Front Hum Neurosci, 13, p. 6.

He, C., Chen, Y., Jian, T., Chen, H., Guo, X., Wang, J., Wu, L., Chen, H. \& Duan, X. (2018) 'Dynamic functional connectivity analysis reveals decreased variability of the default-mode network in developing autistic brain', Autism Res, 11(11), pp. 1479-1493.

Hindriks, R., Adhikari, M. H., Murayama, Y., Ganzetti, M., Mantini, D., Logothetis, N. K. \& Deco, G. (2016) 'Can sliding-window correlations reveal dynamic functional connectivity in resting-state fMRI?', Neuroimage, 127, pp. 242-256.

Hou, W., Sours Rhodes, C., Jiang, L., Roys, S., Zhuo, J., JaJa, J. \& Gullapalli, R. P. (2019) 'Dynamic Functional Network Analysis in Mild Traumatic Brain Injury', Brain Connect, 9(6), pp. 475-487.

Huang, M., Zhou, F., Wu, L., Wang, B., Guo, L., Zhao, Y., Wan, H., Li, F., Zeng, X. \& Gong, H. (2019) 'White matter lesion loads associated with dynamic functional connectivity within attention network in patients with relapsing-remitting multiple sclerosis', J Clin Neurosci, 65, pp. 59-65.

Hutchison, R. M. \& Morton, J. B. (2015) 'Tracking the Brain's Functional Coupling Dynamics over Development', J Neurosci, 35(17), pp. 6849-6859.

Hutchison, R. M., Womelsdorf, T., Allen, E. A., Bandettini, P. A., Calhoun, V. D., Corbetta, M., Della Penna, S., Duyn, J. H., Glover, G. H., Gonzalez-Castillo, J., Handwerker, D. A., Keilholz, S., Kiviniemi, V., Leopold, D. A., de Pasquale, F., Sporns, O., Walter, M. \& Chang, C. (2013) 'Dynamic functional connectivity: promise, issues, and interpretations', Neuroimage, 80, pp. 360-378.

Iraji, A., Deramus, T. P., Lewis, N., Yaesoubi, M., Stephen, J. M., Erhardt, E., Belger, A., Ford, J. M., McEwen, S., Mathalon, D. H., Mueller, B. A., Pearlson, G. D., Potkin, S. G., Preda, A., Turner, J. A., Vaidya, J. G., van Erp, T. G. M. \& Calhoun, V. D. (2019a) 'The spatial chronnectome reveals a dynamic interplay between functional segregation and integration', Hum Brain Mapp, 40(10), pp. 3058-3077.

Iraji, A., Fu, Z., Damaraju, E., DeRamus, T. P., Lewis, N., Bustillo, J. R., Lenroot, R. K., Belger, A., Ford, J. M., McEwen, S., Mathalon, D. H., Mueller, B. A., Pearlson, G. D., Potkin, S. G., Preda, A., Turner, J. A., Vaidya, J. G., van Erp, T. G. M. \& Calhoun, V. D. (2019b) 'Spatial dynamics within and between brain functional domains: A hierarchical approach to study time-varying brain function', Hum Brain Mapp, 40(6), pp. 1969-1986.

Iraji, A., Miller, R., Adali, T. \& Calhoun, V. D. (2020) 'Space: A Missing Piece of the Dynamic Puzzle', Trends Cogn Sci, 24(2), pp. 135-149.

Jafri, M. J., Pearlson, G. D., Stevens, M. \& Calhoun, V. D. (2008) 'A method for functional network connectivity among spatially independent resting-state components in schizophrenia', Neuroimage, 39(4), pp. 1666-1681.

Jeong, S. O., Pae, C. \& Park, H. J. (2016) 'Connectivity-based change point detection for large-size functional networks', Neuroimage, 143, pp. 353-363.

Jin, C., Jia, H., Lanka, P., Rangaprakash, D., Li, L., Liu, T., Hu, X. \& Deshpande, G. (2017) 'Dynamic brain connectivity is a better predictor of PTSD than static connectivity', Hum Brain Mapp, 38(9), pp. 4479-4496.

Jones, D. T., Vemuri, P., Murphy, M. C., Gunter, J. L., Senjem, M. L., Machulda, M. M., Przybelski, S. A., Gregg, B. E., Kantarci, K., Knopman, D. S., Boeve, B. F., Petersen, R. C. \& Jack, C. R., Jr. (2012) 'Non-stationarity in the "resting brain's" modular architecture', PLoS One, 7(6), p. e39731. 
Karahanoglu, F. I., Caballero-Gaudes, C., Lazeyras, F. \& Van de Ville, D. (2013) 'Total activation: fMRI deconvolution through spatio-temporal regularization', Neuroimage, 73, pp. 121-134.

Karahanoglu, F. I. \& Van De Ville, D. (2015) 'Transient brain activity disentangles fMRI resting-state dynamics in terms of spatially and temporally overlapping networks', Nat Commun, 6, p. 7751.

Keilholz, S., Caballero-Gaudes, C., Bandettini, P., Deco, G. \& Calhoun, V. (2017) 'Time-Resolved Resting-State Functional Magnetic Resonance Imaging Analysis: Current Status, Challenges, and New Directions', Brain Connect, 7(8), pp. 465-481.

Klugah-Brown, B., Luo, C., He, H., Jiang, S., Armah, G. K., Wu, Y., Li, J., Yin, W. \& Yao, D. (2019) 'Altered Dynamic Functional Network Connectivity in Frontal Lobe Epilepsy', Brain Topogr, 32(3), pp. 394-404.

Kucyi, A. (2018) 'Just a thought: How mind-wandering is represented in dynamic brain connectivity', Neuroimage, 180(Pt B), pp. 505-514.

Kucyi, A., Hove, M. J., Esterman, M., Hutchison, R. M. \& Valera, E. M. (2017) 'Dynamic Brain Network Correlates of Spontaneous Fluctuations in Attention', Cereb Cortex, 27(3), pp. 1831-1840.

Laumann, T. O., Snyder, A. Z., Mitra, A., Gordon, E. M., Gratton, C., Adeyemo, B., Gilmore, A. W., Nelson, S. M., Berg, J. J., Greene, D. J., McCarthy, J. E., Tagliazucchi, E., Laufs, H., Schlaggar, B. L., Dosenbach, N. U. F. \& Petersen, S. E. (2017) 'On the Stability of BOLD fMRI Correlations', Cereb Cortex, 27(10), pp. 4719-4732.

Leonardi, N., Shirer, W. R., Greicius, M. D. \& Van De Ville, D. (2014) 'Disentangling dynamic networks: Separated and joint expressions of functional connectivity patterns in time', Hum Brain Mapp, 35(12), pp. 5984-5995.

Leonardi, N. \& Van De Ville, D. (2015) 'On spurious and real fluctuations of dynamic functional connectivity during rest', Neuroimage, 104, pp. 430-436.

Li, X., Zhu, D., Jiang, X., Jin, C., Zhang, X., Guo, L., Zhang, J., Hu, X., Li, L. \& Liu, T. (2014) 'Dynamic functional connectomics signatures for characterization and differentiation of PTSD patients', Hum Brain Mapp, 35(4), pp. 1761-1778.

Lim, J., Teng, J., Patanaik, A., Tandi, J. \& Massar, S. A. A. (2018) 'Dynamic functional connectivity markers of objective trait mindfulness', Neuroimage, 176, pp. 193-202.

Lin, S. J., Vavasour, I., Kosaka, B., Li, D. K. B., Traboulsee, A., MacKay, A. \& McKeown, M. J. (2018) 'Education, and the balance between dynamic and stationary functional connectivity jointly support executive functions in relapsing-remitting multiple sclerosis', Hum Brain Mapp, 39(12), pp. 5039-5049.

Lindquist, M. A., Xu, Y., Nebel, M. B. \& Caffo, B. S. (2014) 'Evaluating dynamic bivariate correlations in resting-state fMRI: a comparison study and a new approach', Neuroimage, 101, pp. 531-546.

Liu, A., Lin, S. J., Mi, T., Chen, X., Chan, P., Wang, Z. J. \& McKeown, M. J. (2018) 'Decreased subregional specificity of the putamen in Parkinson's Disease revealed by dynamic connectivity-derived parcellation', Neuroimage Clin, 20, pp. 1163-1175.

Liu, F., Wang, Y., Li, M., Wang, W., Li, R., Zhang, Z., Lu, G. \& Chen, H. (2017) 'Dynamic functional network connectivity in idiopathic generalized epilepsy with generalized tonic-clonic seizure', Hum Brain Mapp, 38(2), pp. 957-973. 
Liu, J., Pearlson, G., Windemuth, A., Ruano, G., Perrone-Bizzozero, N. I. \& Calhoun, V. (2009) 'Combining fMRI and SNP data to investigate connections between brain function and genetics using parallel ICA', Hum Brain Mapp, 30(1), pp. 241-255.

Liu, X., Chang, C. \& Duyn, J. H. (2013) 'Decomposition of spontaneous brain activity into distinct fMRI co-activation patterns', Front Syst Neurosci, 7, p. 101.

Liu, X. \& Duyn, J. H. (2013) 'Time-varying functional network information extracted from brief instances of spontaneous brain activity', Proc Natl Acad Sci U S A, 110(11), pp. 4392-4397.

Lurie, D. J., Kessler, D., Bassett, D. S., Betzel, R. F., Breakspear, M., Kheilholz, S., Kucyi, A., Liégeois, R., Lindquist, M. A., McIntosh, A. R., Poldrack, R. A., Shine, J. M., Thompson, W. H., Bielczyk, N. Z., Douw, L., Kraft, D., Miller, R. L., Muthuraman, M., Pasquini, L., Razi, A., Vidaurre, D., Xie, H. \& Calhoun, V. D. (2020) 'Questions and controversies in the study of time-varying functional connectivity in resting fMRI', Network Neuroscience, 4(1), pp. 30-69.

Ma, S., Calhoun, V. D., Phlypo, R. \& Adali, T. (2014) 'Dynamic changes of spatial functional network connectivity in healthy individuals and schizophrenia patients using independent vector analysis', Neuroimage, 90, pp. 196-206.

Madhyastha, T. M., Askren, M. K., Boord, P. \& Grabowski, T. J. (2015) 'Dynamic connectivity at rest predicts attention task performance', Brain Connect, 5(1), pp. 45-59.

Majeed, W., Magnuson, M., Hasenkamp, W., Schwarb, H., Schumacher, E. H., Barsalou, L. \& Keilholz, S. D. (2011) 'Spatiotemporal dynamics of low frequency BOLD fluctuations in rats and humans', Neuroimage, 54(2), pp. 1140-1150.

Marusak, H. A., Elrahal, F., Peters, C. A., Kundu, P., Lombardo, M. V., Calhoun, V. D., Goldberg, E. K., Cohen, C., Taub, J. W. \& Rabinak, C. A. (2018) 'Mindfulness and dynamic functional neural connectivity in children and adolescents', Behav Brain Res, 336, pp. 211-218.

Matsui, T., Murakami, T. \& Ohki, K. (2016) 'Transient neuronal coactivations embedded in globally propagating waves underlie resting-state functional connectivity', Proc Natl Acad Sci U S A, 113(23), pp. 6556-6561.

Matsui, T., Murakami, T. \& Ohki, K. (2019) 'Neuronal Origin of the Temporal Dynamics of Spontaneous BOLD Activity Correlation', Cereb Cortex, 29(4), pp. 1496-1508.

Miller, R. L., Abrol, A., Adali, T., Levin-Schwarz, Y. \& Calhoun, V. D. (2018) 'Resting-State fMRI Dynamics and Null Models: Perspectives, Sampling Variability, and Simulations', Front Neurosci, 12, p. 551.

Miller, R. L., Yaesoubi, M., Turner, J. A., Mathalon, D., Preda, A., Pearlson, G., Adali, T. \& Calhoun, V. D. (2016) 'Higher Dimensional Meta-State Analysis Reveals Reduced Resting fMRI Connectivity Dynamism in Schizophrenia Patients', PLoS One, 11(3), p. e0149849.

Muller, L., Chavane, F., Reynolds, J. \& Sejnowski, T. J. (2018) 'Cortical travelling waves: mechanisms and computational principles', Nat Rev Neurosci, 19(5), pp. 255-268.

Ou, J., Lian, Z., Xie, L., Li, X., Wang, P., Hao, Y., Zhu, D., Jiang, R., Wang, Y., Chen, Y., Zhang, J. \& Liu, T. (2014) 'Atomic dynamic functional interaction patterns for characterization of ADHD', Hum Brain Mapp, 35(10), pp. 5262-5278. 
Plis, S. M., Amin, M. F., Chekroud, A., Hjelm, D., Damaraju, E., Lee, H. J., Bustillo, J. R., Cho, K., Pearlson, G. D. \& Calhoun, V. D. (2018) 'Reading the (functional) writing on the (structural) wall: Multimodal fusion of brain structure and function via a deep neural network based translation approach reveals novel impairments in schizophrenia', Neuroimage, 181, pp. 734-747.

Plis, S. M., Sarwate, A. D., Wood, D., Dieringer, C., Landis, D., Reed, C., Panta, S. R., Turner, J. A., Shoemaker, J. M., Carter, K. W., Thompson, P., Hutchison, K. \& Calhoun, V. D. (2016) 'COINSTAC: A Privacy Enabled Model and Prototype for Leveraging and Processing Decentralized Brain Imaging Data', Front Neurosci, 10, p. 365.

Premi, E., Calhoun, V. D., Diano, M., Gazzina, S., Cosseddu, M., Alberici, A., Archetti, S., Paternico, D., Gasparotti, R., van Swieten, J., Galimberti, D., Sanchez-Valle, R., Laforce, R., Jr., Moreno, F., Synofzik, M., Graff, C., Masellis, M., Tartaglia, M. C., Rowe, J., Vandenberghe, R., Finger, E., Tagliavini, F., de Mendonca, A., Santana, I., Butler, C., Ducharme, S., Gerhard, A., Danek, A., Levin, J., Otto, M., Frisoni, G., Cappa, S., Sorbi, S., Padovani, A., Rohrer, J. D. \& Borroni, B. (2019) 'The inner fluctuations of the brain in presymptomatic Frontotemporal Dementia: The chronnectome fingerprint', Neuroimage, 189, pp. 645-654.

Preti, M. G., Bolton, T. A. \& Van De Ville, D. (2017) 'The dynamic functional connectome: State-of-theart and perspectives', Neuroimage, 160, pp. 41-54.

Qi, S., Sui, J., Chen, J., Liu, J., Jiang, R., Silva, R., Iraji, A., Damaraju, E., Salman, M., Lin, D., Fu, Z., Zhi, D., Turner, J. A., Bustillo, J., Ford, J. M., Mathalon, D. H., Voyvodic, J., McEwen, S., Preda, A., Belger, A., Potkin, S. G., Mueller, B. A., Adali, T. \& Calhoun, V. D. (2019) 'Parallel group ICA+ICA: Joint estimation of linked functional network variability and structural covariation with application to schizophrenia', Hum Brain Mapp, 40(13), pp. 3795-3809.

Qin, J., Chen, S. G., Hu, D., Zeng, L. L., Fan, Y. M., Chen, X. P. \& Shen, H. (2015) 'Predicting individual brain maturity using dynamic functional connectivity', Front Hum Neurosci, 9, p. 418.

Qiu, L., Xia, M., Cheng, B., Yuan, L., Kuang, W., Bi, F., Ai, H., Gu, Z., Lui, S., Huang, X., He, Y. \& Gong, Q. (2018) 'Abnormal dynamic functional connectivity of amygdalar subregions in untreated patients with first-episode major depressive disorder', J Psychiatry Neurosci, 43(4), pp. 262-272.

Quevenco, F. C., Preti, M. G., van Bergen, J. M., Hua, J., Wyss, M., Li, X., Schreiner, S. J., Steininger, S. C., Meyer, R., Meier, I. B., Brickman, A. M., Leh, S. E., Gietl, A. F., Buck, A., Nitsch, R. M., Pruessmann, K. P., van Zijl, P. C., Hock, C., Van De Ville, D. \& Unschuld, P. G. (2017) 'Memory performance-related dynamic brain connectivity indicates pathological burden and genetic risk for Alzheimer's disease', Alzheimers Res Ther, 9(1), p. 24.

Rashid, B., Blanken, L. M. E., Muetzel, R. L., Miller, R., Damaraju, E., Arbabshirani, M. R., Erhardt, E. B., Verhulst, F. C., van der Lugt, A., Jaddoe, V. W. V., Tiemeier, H., White, T. \& Calhoun, V. (2018) 'Connectivity dynamics in typical development and its relationship to autistic traits and autism spectrum disorder', Hum Brain Mapp, 39(8), pp. 3127-3142.

Sadaghiani, S., Poline, J. B., Kleinschmidt, A. \& D'Esposito, M. (2015) 'Ongoing dynamics in large-scale functional connectivity predict perception', Proc Natl Acad Sci U S A, 112(27), pp. 8463-8468.

Sakoglu, U., Pearlson, G. D., Kiehl, K. A., Wang, Y. M., Michael, A. M. \& Calhoun, V. D. (2010) 'A method for evaluating dynamic functional network connectivity and task-modulation: application to schizophrenia', MAGMA, 23(5-6), pp. 351-366. 
Salman, M. S., Vergara, V. M., Damaraju, E. \& Calhoun, V. D. (2019) 'Decreased Cross-Domain Mutual Information in Schizophrenia From Dynamic Connectivity States', Front Neurosci, 13, p. 873.

Schumacher, J., Peraza, L. R., Firbank, M., Thomas, A. J., Kaiser, M., Gallagher, P., O'Brien, J. T., Blamire, A. M. \& Taylor, J. P. (2019) 'Dynamic functional connectivity changes in dementia with Lewy bodies and Alzheimer's disease', Neuroimage Clin, 22, p. 101812.

Shi, L., Sun, J., Wu, X., Wei, D., Chen, Q., Yang, W., Chen, H. \& Qiu, J. (2018) 'Brain networks of happiness: dynamic functional connectivity among the default, cognitive and salience networks relates to subjective well-being', Soc Cogn Affect Neurosci, 13(8), pp. 851-862.

Shine, J. M., Bissett, P. G., Bell, P. T., Koyejo, O., Balsters, J. H., Gorgolewski, K. J., Moodie, C. A. \& Poldrack, R. A. (2016a) 'The Dynamics of Functional Brain Networks: Integrated Network States during Cognitive Task Performance', Neuron, 92(2), pp. 544-554.

Shine, J. M., Koyejo, O. \& Poldrack, R. A. (2016b) 'Temporal metastates are associated with differential patterns of time-resolved connectivity, network topology, and attention', Proc Natl Acad Sci U S A, 113(35), pp. 9888-9891.

Smith, D. M., Zhao, Y., Keilholz, S. D. \& Schumacher, E. H. (2018) 'Investigating the Intersession Reliability of Dynamic Brain-State Properties', Brain Connect, 8(5), pp. 255-267.

Sui, J., Adali, T., Pearlson, G. D., Clark, V. P. \& Calhoun, V. D. (2009) 'A method for accurate group difference detection by constraining the mixing coefficients in an ICA framework', Hum Brain Mapp, 30(9), pp. 2953-2970.

Sun, Y., Collinson, S. L., Suckling, J. \& Sim, K. (2019) 'Dynamic Reorganization of Functional Connectivity Reveals Abnormal Temporal Efficiency in Schizophrenia', Schizophr Bull, 45(3), pp. 659669.

Tagliazucchi, E., Balenzuela, P., Fraiman, D. \& Chialvo, D. R. (2012) 'Criticality in large-scale brain FMRI dynamics unveiled by a novel point process analysis', Front Physiol, 3, p. 15.

Tagliazucchi, E., Balenzuela, P., Fraiman, D., Montoya, P. \& Chialvo, D. R. (2011) 'Spontaneous BOLD event triggered averages for estimating functional connectivity at resting state', Neurosci Lett, $\mathbf{4 8 8}(2)$, pp. 158-163.

Tagliazucchi, E., Siniatchkin, M., Laufs, H. \& Chialvo, D. R. (2016) 'The Voxel-Wise Functional Connectome Can Be Efficiently Derived from Co-activations in a Sparse Spatio-Temporal Point-Process', Front Neurosci, 10, p. 381.

Thompson, G. J. (2018) 'Neural and metabolic basis of dynamic resting state fMRI', Neuroimage, 180(Pt B), pp. 448-462.

Thompson, G. J., Magnuson, M. E., Merritt, M. D., Schwarb, H., Pan, W. J., McKinley, A., Tripp, L. D., Schumacher, E. H. \& Keilholz, S. D. (2013a) 'Short-time windows of correlation between large-scale functional brain networks predict vigilance intraindividually and interindividually', Hum Brain Mapp, 34(12), pp. 3280-3298.

Thompson, G. J., Merritt, M. D., Pan, W. J., Magnuson, M. E., Grooms, J. K., Jaeger, D. \& Keilholz, S. D. (2013b) 'Neural correlates of time-varying functional connectivity in the rat', Neuroimage, 83, pp. 826836. 
Thompson, W. H., Richter, C. G., Plaven-Sigray, P. \& Fransson, P. (2018) 'Simulations to benchmark time-varying connectivity methods for fMRI', PLoS Comput Biol, 14(5), p. e1006196.

Tu, Y., Fu, Z., Zeng, F., Maleki, N., Lan, L., Li, Z., Park, J., Wilson, G., Gao, Y., Liu, M., Calhoun, V., Liang, F. \& Kong, J. (2019) 'Abnormal thalamocortical network dynamics in migraine', Neurology, 92(23), pp. e2706-e2716.

van der Horn, H. J., Vergara, V. M., Espinoza, F. A., Calhoun, V. D., Mayer, A. R. \& van der Naalt, J. (2019) 'Functional outcome is tied to dynamic brain states after mild to moderate traumatic brain injury', Hum Brain Mapp.

Vergara, V. M., Abrol, A. \& Calhoun, V. D. (2019) 'An average sliding window correlation method for dynamic functional connectivity', Hum Brain Mapp, 40(7), pp. 2089-2103.

Vergara, V. M., Mayer, A. R., Damaraju, E. \& Calhoun, V. D. (2017) 'The effect of preprocessing in dynamic functional network connectivity used to classify mild traumatic brain injury', Brain Behav, 7(10), p. e00809.

Vergara, V. M., Mayer, A. R., Kiehl, K. A. \& Calhoun, V. D. (2018) 'Dynamic functional network connectivity discriminates mild traumatic brain injury through machine learning', Neuroimage Clin, 19, pp. 30-37.

Vergara, V. M., Salman, M., Abrol, A., Espinoza, F. A. \& Calhoun, V. D. (2020) 'Determining the number of states in dynamic functional connectivity using cluster validity indexes', J Neurosci Methods, 337, p. 108651.

Vidaurre, D., Smith, S. M. \& Woolrich, M. W. (2017) 'Brain network dynamics are hierarchically organized in time', Proc Natl Acad Sci U S A, 114(48), pp. 12827-12832.

Xu, H., Su, J., Qin, J., Li, M., Zeng, L. L., Hu, D. \& Shen, H. (2018) 'Impact of global signal regression on characterizing dynamic functional connectivity and brain states', Neuroimage, 173, pp. 127-145.

Xu, Y. \& Lindquist, M. A. (2015) 'Dynamic connectivity detection: an algorithm for determining functional connectivity change points in fMRI data', Front Neurosci, 9, p. 285.

Yaesoubi, M., Adali, T. \& Calhoun, V. D. (2018) 'A window-less approach for capturing time-varying connectivity in fMRI data reveals the presence of states with variable rates of change', Hum Brain Mapp, 39(4), pp. 1626-1636.

Yaesoubi, M., Allen, E. A., Miller, R. L. \& Calhoun, V. D. (2015a) 'Dynamic coherence analysis of resting fMRI data to jointly capture state-based phase, frequency, and time-domain information', Neuroimage, 120, pp. 133-142.

Yaesoubi, M., Miller, R. L., Bustillo, J., Lim, K. O., Vaidya, J. \& Calhoun, V. D. (2017) 'A joint timefrequency analysis of resting-state functional connectivity reveals novel patterns of connectivity shared between or unique to schizophrenia patients and healthy controls', Neuroimage Clin, 15, pp. 761-768.

Yaesoubi, M., Miller, R. L. \& Calhoun, V. D. (2015b) 'Mutually temporally independent connectivity patterns: a new framework to study the dynamics of brain connectivity at rest with application to explain group difference based on gender', Neuroimage, 107, pp. 85-94.

Yang, Z., Craddock, R. C., Margulies, D. S., Yan, C. G. \& Milham, M. P. (2014) 'Common intrinsic connectivity states among posteromedial cortex subdivisions: Insights from analysis of temporal dynamics', Neuroimage, 93 Pt 1, pp. 124-137. 
Yue, J. L., Li, P., Shi, L., Lin, X., Sun, H. Q. \& Lu, L. (2018) 'Enhanced temporal variability of amygdalafrontal functional connectivity in patients with schizophrenia', Neuroimage Clin, 18, pp. 527-532.

Zalesky, A. \& Breakspear, M. (2015) 'Towards a statistical test for functional connectivity dynamics', Neuroimage, 114, pp. 466-470.

Zalesky, A., Fornito, A., Cocchi, L., Gollo, L. L. \& Breakspear, M. (2014) 'Time-resolved resting-state brain networks', Proc Natl Acad Sci US A, 111(28), pp. 10341-10346.

Zhang, C., Baum, S. A., Adduru, V. R., Biswal, B. B. \& Michael, A. M. (2018a) 'Test-retest reliability of dynamic functional connectivity in resting state fMRI', Neuroimage, 183, pp. 907-918.

Zhang, W., Li, S., Wang, X., Gong, Y., Yao, L., Xiao, Y., Liu, J., Keedy, S. K., Gong, Q., Sweeney, J. A. \& Lui, S. (2018b) 'Abnormal dynamic functional connectivity between speech and auditory areas in schizophrenia patients with auditory hallucinations', Neuroimage Clin, 19, pp. 918-924.

Zhi, D., Calhoun, V. D., Lv, L., Ma, X., Ke, Q., Fu, Z., Du, Y., Yang, Y., Yang, X., Pan, M., Qi, S., Jiang, R., Yu, Q. \& Sui, J. (2018) 'Aberrant Dynamic Functional Network Connectivity and Graph Properties in Major Depressive Disorder', Front Psychiatry, 9, p. 339. 